\title{
GEOMETRY BEHIND CHORDAL LOEWNER CHAINS
}

\author{
MANUEL D. CONTRERAS ${ }^{\dagger}$, SANTIAGO DÍAZ-MADRIGAL ${ }^{\dagger}$, AND PAVEL GUMENYUK $^{\ddagger}$
}

\begin{abstract}
Loewner Theory is a deep technique in Complex Analysis affording a basis for many further important developments such as the proof of famous Bieberbach's conjecture and well-celebrated Schramm's Stochastic Loewner Evolution (SLE). It provides analytic description of expanding domains dynamics in the plane. Two cases have been developed in the classical theory, namely the radial and the chordal Loewner evolutions, referring to the associated families of holomorphic self-mappings being normalized at an internal or boundary point of the reference domain, respectively. Recently there has been introduced a new approach [9, 10, 17] bringing together, and containing as quite special cases, radial and chordal variants of Loewner Theory. In the framework of this approach we address the question what kind of systems of simply connected domains can be described by means of Loewner chains of chordal type. As an answer to this question we establish a necessary and sufficient condition for a set of simply connected domains to be the range of a generalized Loewner chain of chordal type. We also provide an easyto-check geometric sufficient condition for that. In addition, we obtain analogous results for the less general case of chordal Loewner evolution considered in [4, 26, 8].
\end{abstract}

\section{CONTENTS}

1. Introduction 2

1.1. Parametric representation 2

1.2. New approach in Loewner Theory

1.3. Problem, definition, and statement of the main results 6

2. Inclusions chains versus Loewner chains 9

3. Sequences of univalent functions $\quad 12$

4. L-admissible families and chordal evolution families $\quad 19$

4.1. Chordally admissible families $\quad 19$

Date: March 2, 2022.

2000 Mathematics Subject Classification. Primary 30C80; Secondary 30D05, 30C35, 34M15.

Key words and phrases. Univalent functions, Loewner chains, Loewner evolution, evolution families, chordal Loewner equation, parametric representation.

$\dagger$ Partially supported by the Ministerio de Ciencia e Innovación and the European Union (FEDER), projects MTM2006-14449-C02-01 and MTM2009-14694-C02-02, by La Consejería de Educación y Ciencia de la Junta de Andalucía and by the ESF Networking Programme "Harmonic and Complex Analysis and its Applications".

$\ddagger$ Partially supported by the ESF Networking Programme "Harmonic and Complex Analysis and its Applications" and the Research Council of Norway. 
4.2. Proof of the Main Theorem $\quad 21$

5. L-admissible families and Goryainov-Ba evolution families $\quad 25$

5.1. Definitions and results 25

5.2. The class $\mathfrak{P}_{0} \quad 28$

5.3. Proof of Theorem 5.5 $\quad 31$

6. Sufficient conditions for chordal admissibility $\quad 32$

7. An example 38

8. Evolution families in the disk algebra $\quad 42$

Acknowledgement $\quad 43$

References

\section{INTRODUCTION}

1.1. Parametric representation. Loewner theory is a deep technique in Geometric Function Theory. In 1923 C. Loewner] published a paper [36] where he introduced the so-called parametric representation of slit mappings, i.e. univalent holomorphic functions mapping the unit disk $\mathbb{D}:=\{z:|z|<1\}$ onto the complex plane minus a slit along a Jordan curve extending to infinity. His original aim was to achieve some progress in the famous Bieberbach conjecture on the sharp upper bounds for the Taylor coefficients of normalized univalent functions. The information about a slit mapping $f$ is encoded in a continuous function $u:[0,+\infty) \rightarrow \mathbb{R}$ in such a way that the function $f$ can be expressed via integrals of the following Loewner $O D E$

$$
\frac{d w}{d t}=-w \frac{e^{i u(t)}+w}{e^{i u(t)}-w}, \quad t \geq 0 ;\left.w\right|_{t=0}=z, \quad z \in \mathbb{D} .
$$

The modern form of the Parametric Representation Method, which provides a tool to represent the whole class $\mathcal{S}$ consisting of all univalent holomorphic functions $f: \mathbb{D} \rightarrow \mathbb{C}$ normalized by the expansion $f(z)=z+a_{2} z^{2}+\ldots$, is due to fundamental contributions of Kufarev [28] and Pommerenke [38]. The Schwarz kernel in the right-hand side of (1.1) is replaced then by an arbitrary holomorphic function $p(w, t)$ measurable in $t$ and satisfying conditions $\operatorname{Re} p(w, t)>0$ and $p(0, t)=1$ for all $w \in \mathbb{D}, t \geq 0$. More details can be found in [39, Chapter 6]. An exposition for the case of slit mappings, essentially the same as originally considered by Loewner, is given in [21, §III.2]. Other good source is [20, Chapter 3] and the monograph [3], which is devoted to the Parametric Representation Method and its applications to extremal problems of Geometric Function Theory.

It is worth mentioning that the Parametric Representation Method, providing one of the main ingredients in the proof of Bieberbach's conjecture given by de Branges [11] in 1984, has however gone far beyond the scope of the initial problem. Two spectacular

\footnotetext{
${ }^{1}$ Charles Loewner was a Czech-American mathematician, also known as Karel Löwner (Czech) and Karl Löwner (German).
} 
examples of its protrusion to other areas of mathematics are the Loewner-Kufarev type equation describing free boundary flow of a viscous fluid in a Hele-Shaw cell [50], see also [27. §1.4.2]; and the highly celebrated Stochastic Loewner Evolution (SLE) introduced in 2000 by Schramm [44] as a powerful tool that led to deep results in the mathematical theory of some 2D lattice models of great importance for Statistical Physics. Recently Markina, Prokhorov, and Vasil'ev [37, 43] have discovered interesting relations between Loewner Theory, Integrable Systems, and Kirillov's representation of the Virasoro algebra, which constitutes a common algebraic skeleton for such topics in Mathematical Physics as the KdV non-linear PDE and String Theory.

Originally Loewner Theory was developed for univalent functions in the unit disk normalized at the origin. Parametric representation of univalent functions in the upper halfplane having hydrodynamical normalization at the point of infinity was developed by Kufarev and his students, see e.g., [30]. The analogue of Loewner ODE (1.1) for this case appeared probably for the first time in papers [29, 41]. Further development in this direction was made in [4, 6, 26, 8].

In modern literature the case of univalent functions normalized at an internal point of the reference domain is referred to as the radial case. The parametric representation of univalent functions with hydrodynamical normalization, in contrast, is marked by the attribute "chordal". The latter of these two cases had been quite underestimated until the already mentioned paper [44] by Schramm, where he used both chordal and radial variants of Loewner Theory to introduce the notion of SLE.

Another mathematical construction closely related to Loewner Theory is one-parametric semigroups of holomorphic functions. We will not discuss it in details in this paper, so we just mention that this construction provides a set of important non-trivial examples for Operator Theory (see, e.g., [47]) and Markov stochastic processes and refer the reader to the monographs [1, 45, 46] for the definition and further details, and to papers [23, 24] where some applications to Probability Theory were developed. An exposition of this topic from the dynamical point of view can be found in [15].

Recently Bracci, Contreras, and Díaz-Madrigal [9, 10, have introduced a new approach in Loewner Theory in order to develop a general construction, which contains, as quite special cases, the radial and chordal Loewner evolutions as well as one-parametric semigroups of holomorphic functions. Now we give a short account on this approach.

1.2. New approach in Loewner Theory. According to the new approach in the Loewner Theory introduced by Bracci, Contreras, and Díaz-Madrigal [9, 10], the essence of this theory can be represented by the relations and interplay between three notions: Loewner chains, evolution families and Herglotz vector fields.

In [9] the following definition of a (generalized) evolution family was given.

Definition 1.1. A family $\left(\varphi_{s, t}\right)_{0 \leq s \leq t<+\infty}$ of holomorphic self-maps of the unit disc is an evolution family of order $d$ with $\bar{d} \in[1,+\infty]$ (in short, an $L^{d}$-evolution family) if 
EF1. $\varphi_{s, s}=i d_{\mathbb{D}}$

EF2. $\varphi_{s, t}=\varphi_{u, t} \circ \varphi_{s, u}$ whenever $0 \leq s \leq u \leq t<+\infty$,

EF3. for all $z \in \mathbb{D}$ and for all $T>0$ there exists a non-negative $k_{z, T} \in L^{d}([0, T], \mathbb{R})$ such that

$$
\left|\varphi_{s, u}(z)-\varphi_{s, t}(z)\right| \leq \int_{u}^{t} k_{z, T}(\xi) d \xi
$$

whenever $0 \leq s \leq u \leq t \leq T$.

We point out that the elements of any evolution family are univalent [9, Corollary 6.3]. The evolution families corresponding to the classical radial case are those satisfying the normalization $\varphi_{0, t}=e^{-t}\left(z+a_{2}(t) z^{2}+\ldots\right), t \geq 0, z \in \mathbb{D}$. Assuming this normalization one can omit condition EF3 in the definition [39, Lemma 6.1]. However, this is obviously not the case in general. Particular notions appeared earlier in the literature, see, e.g., $[22,25,[26,[8,18]$.

Generalized evolution families can be seen as solutions to the following initial value problem

$$
\left\{\begin{array}{l}
\frac{d w}{d t}=G(w, t), \quad t \in[s,+\infty), \\
\left.w\right|_{t=s}=z,
\end{array}\right.
$$

where $G(z, t)$ is a Herglotz vector field. For the definition of Herglotz vector fields and further details we refer the reader to [9]. Here we only mention the fact [9, Theorem 4.8] that Herglotz vector fields can be characterized by the representation

$$
G(z, t)=(\tau(t)-z)(1-\overline{\tau(t)} z) p(z, t)
$$

where $\tau:[0,+\infty) \rightarrow \overline{\mathbb{D}}$ is a measurable function and $p: \mathbb{D} \times[0, \infty) \rightarrow \mathbb{C}$ is a function with non-negative real part, holomorphic in $z$ and locally integrable in $t$. The classical radial case corresponds to $\tau \equiv 0$ and $p(z, t)$ satisfying $p(0, t)=1, t \geq 0$. If we consider the chordal case in the unit disk, then $\tau=1$. More generally, it follows from [9, Thereom 6.7] and the proof of [9, Thereom 7.1] that all non-identical elements of an evolution family have the same Denjoy-Wolff point $\tau_{0}$ if and only if for the corresponding Herglotz vector field we have $\tau \equiv \tau_{0}$. This is the reason for the following definition.

Definition 1.2. Let $\left(\varphi_{s, t}\right)$ be an evolution family. Suppose that all non-identical elements of $\left(\varphi_{s, t}\right)$ share the same Denjoy-Wolff point $\tau_{0} \in \overline{\mathbb{D}}$, i.e., $\varphi_{s, t}\left(\tau_{0}\right)=\tau_{0}$ and $\left|\varphi_{s, t}^{\prime}\left(\tau_{0}\right)\right| \leq 1$ for all $s \geq 0$ and $t \geq s$, where $\varphi_{s, t}\left(\tau_{0}\right)$ and $\varphi_{s, t}^{\prime}\left(\tau_{0}\right)$ are to be understood as the corresponding angular limits if $\tau_{0} \in \mathbb{T}:=\partial \mathbb{D}$. We will say that $\left(\varphi_{s, t}\right)$ is a radial evolution family if $\tau_{0} \in \mathbb{D}$. Otherwise, if $\tau_{0} \in \mathbb{T}$, we will call $\left(\varphi_{s, t}\right)$ a chordal evolution family.

By means of Möbius transformations we can always assume that $\tau_{0}=0$ in the case of a radial evolution family and that $\tau_{0}=1$ in the case of a chordal evolution family.

The general version of the third fundamental notion in Loewner Theory, the notion of generalized Loewner chains was given in [17]. 
Definition 1.3. A family $\left(f_{t}\right)_{0 \leq t<+\infty}$ of holomorphic maps of the unit disc $\mathbb{D}$ is called a Loewner chain of order $d$ with $\bar{d} \in[1,+\infty]$ (in short, an $L^{d}$-Loewner chain) if

LC1. each function $f_{t}: \mathbb{D} \rightarrow \mathbb{C}$ is univalent,

LC2. $f_{s}(\mathbb{D}) \subset f_{t}(\mathbb{D})$ for all $s \geq 0$ and $t \geq s$,

LC3. for any compact set $K \subset \mathbb{D}$ and any $T>0$ there exists a non-negative function $k_{K, T} \in L^{d}([0, T], \mathbb{R})$ such that

$$
\left|f_{s}(z)-f_{t}(z)\right| \leq \int_{s}^{t} k_{K, T}(\xi) d \xi
$$

for all $z \in K$ and all $0 \leq s \leq t \leq T$.

A Loewner chain $\left(f_{t}\right)$ is said to be normalized if $f_{0}(0)=0$ and $f_{0}^{\prime}(0)=1$ (notice that we only normalize the function $f_{0}$ ).

We note that the classical notion of (radial) Loewner chain is recovered if one replaces condition LC3 by the requirement that $f_{t}$ should satisfy the following normalization: $f_{t}(z)=e^{t}\left(z+c_{2}(t) z^{2}+\ldots\right)$.

In [17] we established a deep relation between evolution families and Loewner chains similar to that taking place in the classical case. These results can be stated in the following three theorems.

Theorem A ([17, Theorem 1.3]). For any Loewner chain $\left(f_{t}\right)$ of order $d \in[1,+\infty]$, if we define

$$
\varphi_{s, t}:=f_{t}^{-1} \circ f_{s}, \quad 0 \leq s \leq t,
$$

then $\left(\varphi_{s, t}\right)$ is an evolution family of the same order $d$. Conversely, for any evolution family $\left(\varphi_{s, t}\right)$ of order $d \in[1,+\infty]$, there exists a Loewner chain $\left(f_{t}\right)$ of the same order $d$ such that the following equality holds

$$
f_{t} \circ \varphi_{s, t}=f_{s}, \quad 0 \leq s \leq t .
$$

Definition 1.4. A Loewner chain $\left(f_{t}\right)$ is said to be associated with an evolution family $\left(\varphi_{s, t}\right)$ if it satisfies (1.3). Another way to express the same fact is to say that $\left(\varphi_{s, t}\right)$ is the evolution family of the Loewner chain $\left(f_{t}\right)$.

In general, given an evolution family $\left(\varphi_{s, t}\right)$, algebraic condition (1.3) does not define a unique Loewner chain. In fact, there can be a plenty of different Loewner chains associated with the same evolution family. The following theorem gives necessary and sufficient conditions for the uniqueness of a normalized Loewner chain associated with a given evolution family.

Theorem B ([17, Theorem 1.6]). Let $\left(\varphi_{s, t}\right)$ be an evolution family. Then there exists a unique normalized Loewner chain $\left(f_{t}\right)$ associated with $\left(\varphi_{s, t}\right)$ such that $\cup_{t \geq 0} f_{t}(\mathbb{D})$ is either an Euclidean disk centered at the origin or the whole complex plane $\mathbb{C}$. Moreover, the following statements are equivalent: 
(i) the family $\left(f_{t}\right)$ is the only normalized Loewner chain associated with the evolution family $\left(\varphi_{s, t}\right)$;

(ii) there exist $z \in \mathbb{D}$ such that

$$
\lim _{t \rightarrow+\infty} \frac{\left|\varphi_{0, t}^{\prime}(z)\right|}{1-\left|\varphi_{0, t}(z)\right|^{2}}=0 ;
$$

(iii) the above limit vanishes for all $z \in \mathbb{D}$;

(iv) $\bigcup_{t \geq 0} f_{t}(\mathbb{D})=\mathbb{C}$.

We call the family $\left(f_{t}\right)$ in the above theorem the standard Loewner chain associated with the evolution family $\left(\varphi_{s, t}\right)$.

The following theorem explains what happens when an associated Loewner chain is not unique. As above we will denote by $\mathcal{S}$ the class of all univalent holomorphic functions $h$ in the unit disk $\mathbb{D}$, normalized by $h(0)=h^{\prime}(0)-1=0$.

Theorem C ([17, Theorem 1.7]). Let $\left(\varphi_{s, t}\right)$ be an evolution family and $\left(f_{t}\right)$ the standard Loewner chain associated with $\left(\varphi_{s, t}\right)$. Suppose that

$$
\Omega:=\bigcup_{t \geq 0} f_{t}(\mathbb{D}) \neq \mathbb{C}
$$

and write $\beta:=\lim _{t \rightarrow+\infty} \frac{\left|\varphi_{0, t}^{\prime}(0)\right|}{1-\left|\varphi_{0, t}(0)\right|^{2}}$ (such a limit always exists). Then $\Omega=\{z:|z|<1 / \beta\}$ and for any other normalized Loewner chain $\left(g_{t}\right)$ associated with the evolution family $\left(\varphi_{s, t}\right)$, there is $h \in \mathcal{S}$ such that

$$
g_{t}(z)=h\left(\beta f_{t}(z)\right) / \beta .
$$

Using the relation between Loewner chains and evolution families we can now introduce the following definition.

Definition 1.5. Let $\left(f_{t}\right)$ be a Loewner chain. Suppose that the functions $\varphi_{s, t}:=f_{t}^{-1} \circ f_{s}$, $t \geq s \geq 0$, form a radial (respectively, chordal) evolution family. In this case we will say that the Loewner chain $\left(f_{t}\right)$ is of radial (respectively, chordal) type.

1.3. Problem, definition, and statement of the main results. The general problem this paper is devoted to study the relation between geometric properties of Loewner chains and analytic properties of their evolution families. More specifically, our aim is to give a complete characterization for the systems of image domains $f_{t}(\mathbb{D})$ generated by Loewner chains whose evolution family belongs to one or another certain class, including that of all radial and chordal evolution families.

As we will see a little bit later, for the case of radial evolution families this problem turns out to be solved by means of simple modifications of some proofs in the classical Loewner Theory. At the same time the chordal case seems to be much harder. The same 
can be said concerning the class of evolution families introduced in [22], which we consider in Section 5 .

To formulate our main results we introduce the following definition.

Definition 1.6. Let $\left(\Omega_{t}\right)_{t \geq 0}$ be a family of simply connected domains $\Omega_{t} \subset \mathbb{C}$ and $\Omega_{t} \neq \mathbb{C}$ for all $t \in[0,+\infty)$. The family $\left(\Omega_{t}\right)_{t \geq 0}$ is said to be a inclusion chain if it satisfies the following conditions:

IC1. $\Omega_{s} \subset \Omega_{t}$ whenever $t \geq s \geq 0$.

IC2. $\Omega_{t_{n}} \rightarrow \Omega_{t_{0}}$ whenever $t_{n} \rightarrow t_{0}$ in the sense of the kernel convergence w.r.t. any point of $\Omega_{0}$.

For the notion of kernel convergence of domains we refer the reader to [21, §II.5] or [39, Section 1.4].

Remark 1.7. We note that, as it follows from the definition of the kernel of a sequence of domains, condition IC2 above can replaced by the two following simple conditions of topological nature:

(i) for each $t \geq 0$ the domain $\Omega_{t}$ is a connected component of the interior of $\bigcap_{s>t} \Omega_{s}$,

(ii) for each $t>0$ the domain $\Omega_{t}$ coincides with $\bigcup_{s \in[0, t)} \Omega_{s}$.

Denote by $r(D, z)$ the conformal radius of a simply connected domain $D$ w.r.t. a point $z$. The following two theorems explain our motivation to introduce the notion of inclusion chain. As far as we know, these theorems have not been stated previously but we think they can be considered as well-known among the specialists. In any case, for the sake of completeness we will sketch their proofs in Section 2.

Theorem 1.8. Let $\left(\Omega_{t}\right)_{t \geq 0}$ be a family of simply connected domains $\Omega_{t} \subset \mathbb{C}$. Suppose that $\Omega_{s} \subset \Omega_{t} \neq \mathbb{C}$ for all $s \geq 0$ and $t \geq s$. Then the following assertions are equivalent:

(1) The family $\left(\Omega_{t}\right)_{t \geq 0}$ is an inclusion chain.

(2) There exists $w_{*} \in \Omega_{0}$ such that the function $t \in[0,+\infty) \mapsto \mu_{w_{*}}(t):=r\left(\Omega_{t}, w_{*}\right)$ is continuous.

(3) For all $w_{*} \in \Omega_{0}$ the function $t \in[0,+\infty) \mapsto \mu_{w_{*}}(t):=r\left(\Omega_{t}, w_{*}\right)$ is non-decreasing and continuous.

Theorem 1.9. Under conditions of Theorem 1.8 the following two assertions are equivalent:

(1) The family $\left(\Omega_{t}\right)_{t \geq 0}$ is a inclusion chain.

(2) There exists a Loewner chain $\left(f_{t}\right)$ such that

$$
\left\{\Omega_{t}: t \geq 0\right\}=\left\{f_{t}(\mathbb{D}): t \geq 0\right\} .
$$

In the proofs of the above two theorems, the Loewner chain that we construct for a given inclusion chain $\left(\Omega_{t}\right)_{t \geq 0}$, is of radial type. This is not surprising, because in [17] (proof of Theorem 3.3) we showed that given an arbitrary Loewner chain $\left(f_{t}\right)$, then there is a 
Loewner chain $\left(g_{t}\right)$ of radial type such that $f_{t}(\mathbb{D})=g_{t}(\mathbb{D})$ for all $t \geq 0$. However, this statement does not hold any more if one requires $\left(g_{t}\right)$ to be a Loewner chain of chordal type instead of that of radial type. This can be easily seen if we consider any inclusion chain $\left(\Omega_{t}\right)$ having the following property: there exists $s, t \geq 0$ such that $\partial \Omega_{s} \cap \partial \Omega_{t}=\emptyset$.

So we address the following problem.

Problem. Characterize all inclusion chains $\left(\Omega_{t}\right)$ which are the ranges of Loewner chains of chordal type, i.e., $\left\{\Omega_{t}: t \geq 0\right\}=\left\{f_{t}(\mathbb{D}): t \geq 0\right\}$ for some Loewner chain $\left(f_{t}\right)$ such that the functions $\varphi_{s, t}=f_{t}^{-1} \circ f_{s}, t \geq s \geq 0$, form a chordal evolution family.

One can regard as known the fact that the class of all inclusion chains satisfying the condition in the above problem contains all "slit" inclusion chains, i.e. inclusion chains obtained by gradual removing a slit (or a finite system of slits) in a simply connected domain, see Section [7. Our main result concerns the general case and can be formulated as follows. Denote by $\mathrm{P}(D)$ the Carathéodory boundary of a domain $D$, i.e. the set of all its prime ends.

Theorem 1.10. Let $\left(\Omega_{t}\right)_{t \geq 0}$ be an inclusion chain. Then the following statements are equivalent:

(1) There exists a prime end $P_{0} \in \mathrm{P}\left(\Omega_{0}\right)$ such that for each $t \geq 0$ the domain $\Omega_{0}$ is embedded into the domain $\Omega_{t}$ conformally at the prime end $P_{0}$.

(2) There is a Loewner chain $\left(f_{t}\right)$ of chordal type such that

$$
\left\{\Omega_{t}: t \geq 0\right\}=\left\{f_{t}(\mathbb{D}): t \geq 0\right\} .
$$

For the notion of domain embedded conformally at a prime end, see Definitions 4.1 and 4.2 in Section 4 .

The above theorem is a direct consequence of the more technical Theorem 4.8. It is worth mentioning that the Loewner chain $\left(f_{t}\right)$ in this theorem can be always assumed to be of order $d=\infty$.

In Section 5 we state and proof a result (Theorem 5.5), which is an analogue of Theorem 1.10 for a subclass of chordal evolution families having some additional regularity, which were considered in [26].

Theorems 1.10 and 5.5 give necessary and sufficient conditions for an inclusion chain to be the range of a Loewner chain of given type. However, in general these conditions might be quite difficult to verify. Therefore in Section [6 we give some easy-to-check geometric sufficient conditions.

Finally in Sections 7 and 8 we consider the question how the boundary behaviour of an evolution family affects the geometry of the associated Loewner chains. It turns out (Proposition (7.3) that there exists an evolution family consisting of functions with continuous extensions to the unit circle such that none of the associated Loewner chains has image domains with locally connected boundaries. At the same time, as we prove in Section 8 , if a Loewner chain $\left(f_{t}\right)$ has the property that $\partial f_{t}(\mathbb{D})$ is locally connected for 
all $t \geq 0$, then all the functions in the evolution family of this Loewner chain can be continuously extended to the unit circle.

It is also worth mentioning that further in the paper we prefer to work with the notion of L-admissible family instead of that of inclusion chain, see Definition 2.4 in the next section. In some cases, however, it obviously makes absolutely no difference and many results hold with words "L-admissible family " replaced by "inclusion chain".

\section{INCLUSIONS CHAINS VERSUS LOEWNER CHAINS}

In this section we will sketch the proofs of Theorems 1.8 and 1.9, Let us start with some auxilliary statements.

Lemma 2.1. Let $\varphi$ be a holomorphic univalent self-mapping of the unit disk $\mathbb{D}$ with $\varphi(0)=0$. Then:

(1) for all $z \in \mathbb{D}$,

$$
|\varphi(z)-z| \leq\left|1-\varphi^{\prime}(0)\right|+\sqrt{1-\left|\varphi^{\prime}(0)\right|^{2}}
$$

(2) if $\varphi^{\prime}(0)>0$, then for all $z \in \mathbb{D}$,

$$
|\varphi(z)-z| \leq 3 \sqrt{1-\varphi^{\prime}(0)} .
$$

Proof. Write $\varphi(z)=\sum_{n=1}^{\infty} a_{n} z^{n}$. Let us recall that

$$
\sum_{n=1}^{\infty} n\left|a_{n}\right|^{2}=m(\varphi(\mathbb{D})) \leq m(\mathbb{D})=1,
$$

where $m(\cdot)$ stands for the normalized Lebesgue measure in the unit disc. Let $\phi(z):=\varphi(z)-a_{1} z$. Therefore

$$
\begin{aligned}
|\varphi(z)-z| & \leq\left|\sum_{n=2}^{\infty} a_{n} z^{n}\right|+\left|1-\varphi^{\prime}(0)\right| \leq\|\phi\|_{H_{\infty}}+\left|1-\varphi^{\prime}(0)\right| \leq\|\phi\|_{H_{2}}+\left|1-\varphi^{\prime}(0)\right| \\
& =\sqrt{\sum_{n=2}^{\infty}\left|a_{n}\right|^{2}+\left|1-\varphi^{\prime}(0)\right| \leq \sqrt{\sum_{n=2}^{\infty} n\left|a_{n}\right|^{2}+\left|1-\varphi^{\prime}(0)\right|}} \\
& \leq \sqrt{1-\left|\varphi^{\prime}(0)\right|^{2}}+\left|1-\varphi^{\prime}(0)\right| .
\end{aligned}
$$

This proves (1). Assertion (2) is now an immediate consequence of (1).

Lemma 2.2. Let $f, g: \mathbb{D} \rightarrow \mathbb{C}$ be two univalent functions such that

(1) $f(0)=g(0)$,

(2) $f(\mathbb{D}) \subset g(\mathbb{D})$,

(3) $f^{\prime}(0), g^{\prime}(0)>0$. 
Then for all $r \in(0,1)$ there exists a constant $C=C(r)>0$ not depending on the functions $f$ and $g$ such that

$$
|f(z)-g(z)| \leq C \sqrt{g^{\prime}(0)\left(g^{\prime}(0)-f^{\prime}(0)\right)}, \quad \text { for all }|z| \leq r .
$$

Proof. Write $\varphi=g^{-1} \circ f$ and fix $r<1$. Notice that $\varphi(D(0, r)) \subseteq D(0, r)$. Take any $z$ with $|z| \leq r$ and denote by $I$ the line segment joining the points $z$ and $\varphi(z)$. Then using the Koebe distortion theorem (see, e.g., [21, §II.4] or [39, Theorem 1.6 on p.21]) we get

$$
\begin{aligned}
|f(z)-g(z)| & =|g(\varphi(z))-g(z)|=\left|\int_{z}^{\varphi(z)} g^{\prime}(\xi) d \xi\right| \leq|\varphi(z)-z| \max _{\xi \in I}\left|g^{\prime}(\xi)\right| \\
& \leq|\varphi(z)-z| g^{\prime}(0) \frac{1+r}{(1-r)^{3}} \leq g^{\prime}(0) \frac{2}{(1-r)^{3}}|\varphi(z)-z| .
\end{aligned}
$$

Now, by the above lemma,

$$
|f(z)-g(z)| \leq g^{\prime}(0) \frac{6}{(1-r)^{3}} \sqrt{1-\varphi^{\prime}(0)}=\frac{6}{(1-r)^{3}} \sqrt{g^{\prime}(0)\left(g^{\prime}(0)-f^{\prime}(0)\right)}
$$

Proof of Theorem 1.8. The fact that (1) implies (2) and (3) is just one of the implications in the Carathéodory kernel theorem (see, e.g., [21, Theorem 1 on p. 55] or [39, Theorem 1.8 on p. 29]). The function $\mu_{w_{*}}$ is non-decreasing for any $w_{*} \in \Omega_{0}$ due to the inclusions $\Omega_{s} \subset$ $\Omega_{t}, 0 \leq s \leq t$. Being clear that (3) implies (2), it remains to prove that (2) implies (1).

Let us fix a sequence of non-negative real numbers $\left(t_{n}\right)$ converging to $t_{0} \in[0,+\infty)$. We have to prove that $\Omega_{t_{n}}$ converges to $\Omega_{t_{0}}$ in the sense of the kernel convergence. By the very definition of conformal radius, there are univalent functions in the unit disk $f_{n}$ and $f$ such that $f_{n}(\mathbb{D})=\Omega_{t_{n}}, f(\mathbb{D})=\Omega_{t_{0}}, f_{n}(0)=f(0)=w_{*}, f_{n}^{\prime}(0)=r\left(\Omega_{t_{n}}, w_{*}\right), f^{\prime}(0)=r\left(\Omega_{t_{0}}, w_{*}\right)$ for all $n$. By hypothesis, $f_{n}^{\prime}(0) \rightarrow f^{\prime}(0)$.

We have to prove that $f_{n}$ converges uniformly on compacta to $f$. Fix $0<r<1$. We may assume that the sequence $\left(t_{n}\right)$ is monotone. Let us assume first that $\left(t_{n}\right)$ is non-decreasing. By Lemma 2.2, there is a constant $C=C(r)$ such that

$$
\left|f(z)-f_{n}(z)\right| \leq C \sqrt{f^{\prime}(0)\left(f^{\prime}(0)-f_{n}^{\prime}(0)\right)}, \quad \text { for all }|z| \leq r .
$$

If the sequence $t_{n}$ is non-increasing we obtain in a similar way that

$$
\left|f(z)-f_{n}(z)\right| \leq C \sqrt{f_{n}^{\prime}(0)\left(f_{n}^{\prime}(0)-f^{\prime}(0)\right)}, \quad \text { for all }|z| \leq r .
$$

In both cases, we conclude that $f_{n}$ converges uniformly on compacta to $f$. The proof of Theorem 1.8 is now finished.

Now we turn to the proof of Theorem 1.9. Before doing this let us consider the following natural question: is it possible to replace the set equality

$$
\left\{\Omega_{t}: t \geq 0\right\}=\left\{f_{t}(\mathbb{D}): t \geq 0\right\}
$$


in Theorem 1.9 by the stronger requirement that $\Omega_{t}=f_{t}(\mathbb{D})$ for every $t>0$ ? The answer turns out to be negative, see Example 2.5 below. The reason for that is explained by the following theorem. Denote by $A C^{d}(X, Y), X \subset \mathbb{R}, d \in[1,+\infty]$, the class of all locally absolutely continuous functions $f: X \rightarrow Y$ such that the derivative $f^{\prime}$ belongs to $L_{\text {loc }}^{d}(X)$.

Theorem 2.3. Let $\left(\Omega_{t}\right)_{t \geq 0}$ be a family of simply connected domains and $d \in[1,+\infty]$. The three statements below are equivalent:

(i) The following two conditions are fulfilled:

AF1. $\Omega_{s} \subset \Omega_{t} \neq \mathbb{C}$ whenever $t \geq s \geq 0$.

AF2. There exists $w_{*} \in \Omega_{0}$ such that the function $\mu_{w_{*}}(t):=r\left(\Omega_{t}, w_{*}\right)$ belongs to $A C^{d}([0,+\infty), \mathbb{R})$.

(ii) The family $\left(\Omega_{t}\right)$ satisfies condition $A F 1$ and the following assertion holds:

AF2'. For all $w_{*} \in \Omega_{0}$ the function $\mu_{w_{*}}(t):=r\left(\Omega_{t}, w_{*}\right)$ is non-decreasing and belongs to $A C^{d}([0,+\infty), \mathbb{R})$.

(iii) There exists a Loewner chain $\left(f_{t}\right)$ of order $d$ such that $f_{t}(\mathbb{D})=\Omega_{t}$ for all $t \geq 0$.

This theorem explains our motivation to introduce the following definition.

Definition 2.4. A family $\left(\Omega_{t}\right)_{t \geq 0}$ of simply connected domains $\Omega_{t} \subset \mathbb{C}$ is said to be an L-admissible family of order $d \in[1,+\infty]$ if it satisfies conditions AF1 and AF2 in Theorem 2.3.

Example 2.5. Take $\gamma:[0,+\infty) \rightarrow[1,+\infty)$ a non-decreasing function which is continuous but not absolutely continuous. For each $t \geq 0$, define $\Omega_{t}:=\gamma(t) \mathbb{D}$. Obviously, $r\left(\Omega_{t}, 0\right)=$ $\gamma(t)$. The increasing family of domains $\left(\Omega_{t}\right)$ is an inclusion chain, but it is not an Ladmissible family and consequently there exist no Loewner chains $\left(f_{t}\right)$ such that $f_{t}(\mathbb{D})=$ $\Omega_{t}$ for all $t \geq 0$.

For the proof of the above theorem we will need the following lemma.

Lemma 2.6. Let $\left(f_{t}\right)$ be a Loewner chain and $\left(\varphi_{s, t}\right)$ its evolution family. Let $z_{0} \in \mathbb{D}$ and $w_{0}:=f_{0}\left(z_{0}\right)$. Then for each $t \geq 0$ the conformal radius $\rho\left(z_{0}, t\right)$ of $f_{t}(\mathbb{D})$ w.r.t. $w_{0}$ equals $\left|f_{0}^{\prime}\left(z_{0}\right)\right| / \beta_{t}\left(z_{0}\right)$, where

$$
\beta_{t}(z):=\frac{\left|\varphi_{0, t}^{\prime}(z)\right|}{1-\left|\varphi_{0, t}(z)\right|^{2}}, \quad z \in \mathbb{D}, t \geq 0 .
$$

Proof. Let $z_{t}:=\varphi_{0, t}\left(z_{0}\right)$ and

$$
\ell_{t}(z)=\frac{z+z_{t}}{1+\overline{z_{t}} z}
$$

The function $g_{t}:=f_{t} \circ \ell_{t}$ maps conformally $\mathbb{D}$ onto $f_{t}(\mathbb{D})$. Moreover, $g_{t}(0)=w_{0}$ because

$$
f_{t} \circ \varphi_{0, t}=f_{0} .
$$

Hence $\rho\left(z_{0}, t\right)=\left|g_{t}^{\prime}(0)\right|$. By the chain rule, from (2.1) it follows that $f_{t}^{\prime}\left(z_{t}\right) \varphi_{0, t}^{\prime}\left(z_{0}\right)=f_{0}^{\prime}\left(z_{0}\right)$. Now the proof can be completed by means of simple computations. 
Proof of Theorem [2.3. First of all let us prove that assertion (i) implies (iii). By the Riemann mapping theorem, for all $t \geq 0$ there is a unique univalent holomorphic function $f_{t}: \mathbb{D} \rightarrow \mathbb{C}$ such that $f_{t}(\mathbb{D})=\Omega_{t}, f_{t}(0)=w_{*}$ and $f_{t}^{\prime}(0)>0$. We will prove that the family $\left(f_{t}\right)$ is a Loewner chain of order $d$.

Write $\varphi_{s, t}(z)=f_{t}^{-1} \circ f_{s}(z)$ for all $z \in \mathbb{D}$ and for all $0 \leq s \leq t$. It is clear that the functions $\varphi_{s, t}$ are univalent and the family $\left(\varphi_{s, t}\right)$ satisfied EF1 and EF2. Since the function $t \mapsto b(t)=\varphi_{0, t}^{\prime}(0)=f_{0}^{\prime}(0) / f_{t}^{\prime}(0)=f_{0}^{\prime}(0) / r\left(\Omega_{t}, w_{*}\right)$ belongs to $A C^{d}([0,+\infty), \mathbb{R})$ and the map $t \mapsto a(t)=\varphi_{0, t}(0)=0$ is constant, by [17, Proposition 2.10], $\left(\varphi_{s, t}\right)$ is an evolution family of order $d$. Finally, since the functions $f_{t}$ are univalent and $f_{t} \circ \varphi_{s, t}=f_{s}$ for all $0 \leq s \leq t$, by [17, Lemma 3.2], the family $\left(f_{t}\right)$ is a Loewner chain of order $d$.

Being trivial that (ii) implies (i), it remains to prove that (iii) implies (ii). So take a Loewner chain $\left(f_{t}\right)$ of order $d$ such that $f_{t}(\mathbb{D})=\Omega_{t}$ for all $t \geq 0$ with associated evolution family given by $\left(\varphi_{s, t}\right)$. By the very definition of Loewner chain, it is clear that the family $\left(\Omega_{t}\right)$ satisfies AF1. Fix $w_{*} \in \Omega_{0}$ and write $\mu_{w_{*}}(t):=r\left(\Omega_{t}, w_{*}\right)$. Clearly, the function $\mu_{w_{*}}$ is non-decreasing. Take $z_{*} \in \mathbb{D}$ such that $w_{*}:=f_{0}\left(z_{*}\right)$ and define $a(t)=$ $\varphi_{0, t}\left(z_{*}\right)$ and $b(t)=\varphi_{0, t}^{\prime}\left(z_{*}\right)$ for all $t \geq 0$. By [17, Proposition 2.10], $a$ and $b$ belong to $A C^{d}([0,+\infty), \mathbb{R})$. Moreover, by Lemma 2.6, we have that $\mu_{w_{*}}(t)=\left|f_{0}^{\prime}\left(z_{0}\right)\right| \frac{1-|a(t)|^{2}}{|b(t)|}$. Finally, a direct computation shows that $\mu_{w_{*}}$ also belongs to $A C^{d}([0,+\infty), \mathbb{R})$. This completes the proof.

Now we can deduce Theorem 1.9 from Theorem 2.3.

Proof of Theorem 1.9. Notice that if we manage to construct a continuous non-decreasing function $h:[0,+\infty) \rightarrow[0,+\infty)$ with $h(0)=0$ and $\lim _{t \rightarrow+\infty} h(t)=+\infty$ such that $\Omega_{h(t)}$ is an L-admissible family of order $d \in[1,+\infty]$, we will finish just by applying Theorem [2.3.

Denote $J:=\mu_{w_{*}}([0,+\infty))$ and take any monotone function $g$ mapping $[0,+\infty)$ onto $J$ that belongs to $A C^{\infty}([0,+\infty), \mathbb{R})$. The function $h$ we are looking for is given by

$$
h(t):=\inf \left\{\theta \geq 0: \mu_{w_{*}}(\theta)=g(t)\right\} .
$$

The proof is now finished.

\section{Sequences of univalent Functions}

In this section we make ready the key tool to solve our problem. We present a result on convergence of sequences of univalent functions in the spirit of the Carathéodory kernel theorem.

When dealing with holomorphic self-mappings having a boundary fixed point it is more convenient to switch from the unit disk to the upper half-plane $\mathbb{H}:=\{z: \operatorname{Im} z>0\}$. It can be made by means of the change of variables $w=H(\zeta)$, where

$$
H(\zeta):=i \frac{1+\zeta}{1-\zeta}
$$


is the so called Cayley map which sends the unit disk $\mathbb{D}$ onto the upper half-plane $\mathbb{H}$, with $H(0)=i$ and $H(1)=\infty$.

We will need the following classical result (see, e.g. [49, Ch. IV §26]).

Theorem D. Let $f: \mathbb{H} \rightarrow \mathbb{H}$ be a holomorphic function. Then

$$
f(z)=c z+\Phi(z), \quad z \in \mathbb{H},
$$

where

$$
c:=\inf _{z \in \mathbb{H}} \frac{\operatorname{Im} f(z)}{\operatorname{Im} z}
$$

and $\Phi: \mathbb{H} \rightarrow \mathbb{H}$ is a holomorphic function such that

$$
\angle \lim _{z \rightarrow \infty} \frac{\Phi(z)}{z}=0
$$

The number $c$ in the above theorem is called the angular derivative of the function $f$ at $\infty$. We will denote this number by $f^{\prime}(\infty)$. A very simple but useful consequence of Theorem $\mathrm{D}$ is the following

Lemma 3.1. Suppose $f: \mathbb{H} \rightarrow \mathbb{H}$ is a holomorphic function and $A$ is a non-negative number. Then the following assertions are equivalent:

(i) the angular derivative of $f$ at $\infty$ equals $A$;

(ii) $\angle \lim _{z \rightarrow \infty} \frac{f(z)}{z}=A$;

(iii) $\lim _{y \rightarrow+\infty} \frac{f(i y)}{i y}=A$;

(iv) $\angle \lim _{z \rightarrow \infty} \frac{\operatorname{lm}(f(z)-A z)}{|z|}=0$;

(v) $\lim _{y \rightarrow+\infty} \frac{\operatorname{Im}(f(i y)-i A y)}{y}=0$;

Proof. First suppose (i) holds. Then $\Phi(z):=f(z)-A z$ satisfies (3.3). It immediately follows that (ii)-(v) take place.

Now let us denote by $c$ the angular derivative of $f$ at $\infty$. Then (ii) $-(\mathrm{v})$ hold with $c$ substituted for $A$. At the same time whenever the function $f$ is fixed, each of these assertions can hold for at most one value of $A$. This proves that (i) is implied by each of assertions (ii)-(v).

Remark 3.2. Another consequence of Theorem $\mathrm{D}$ is the following well-known assertion: if $\varphi \in \operatorname{Hol}(\mathbb{D}, \mathbb{D})$ and $\exists \angle \lim _{z \rightarrow 1} \varphi(z)=1$, then there exists the angular derivative

$$
\varphi^{\prime}(1):=\angle \lim _{z \rightarrow 1} \frac{\varphi(z)-1}{z-1},
$$


which can be finite or infinite. If $\varphi^{\prime}(1) \neq \infty$, then $\varphi^{\prime}(1)>0$.

It is easy to see that if $f \in \operatorname{Hol}(\mathbb{H}, \mathbb{H})$ and $f^{\prime}(\infty) \neq 0$, then $\angle \lim _{z \rightarrow \infty} f(z)=\infty$. The latter means that $f$ has a boundary fixed point at $z=\infty$. If $f^{\prime}(\infty)=1$, this boundary fixed point is said to be parabolic. Let us denote by $\mathfrak{P}$ the class of all univalent functions from $\operatorname{Hol}(\mathbb{H}, \mathbb{H})$ that have a parabolic boundary fixed point at $z=\infty$,

$$
\mathfrak{P}:=\left\{f \in \operatorname{Hol}(\mathbb{H}, \mathbb{H}): f^{\prime}(\infty)=1, f \text { is univalent in } \mathbb{H}\right\} \text {. }
$$

Similarly, by $\mathcal{P}$ we will denote the class of all univalent functions $\varphi \in \operatorname{Hol}(\mathbb{D}, \mathbb{D})$ satisfying equalities $\varphi(1)=1$ and $\varphi^{\prime}(1)=1$ in the angular sense. Equivalently,

$$
\mathcal{P}:=\left\{H^{-1} \circ f \circ H: f \in \mathfrak{P}\right\} .
$$

Remark 3.3. ¿From Theorem $\mathrm{D}$ it follows that $\operatorname{Im}(f(z)-z) \geq 0, z \in \mathbb{H}$, for all $f \in \mathfrak{P}$, a fact that will be used on numerous occasions. The equality can happen only for functions $f(z)=z+C$, where $C \in \mathbb{R}$ is a constant.

We will also use on several occasions the following theorem due to Koebe (see, e.g., [21, Theorem 1, §II.3] or [40, Proposition 2.14]). A curve $\Gamma:[0,1) \rightarrow \overline{\mathbb{C}}$ is said to land at a point $p$ if there exists $\lim _{x \rightarrow 1-} \Gamma(x)=p$. By $\partial_{\infty} E$ we will denote the boundary of a set $E$ in the Riemann sphere $\overline{\mathbb{C}}$.

Theorem E. Let $f$ be a univalent mapping of $\mathbb{D}$ onto a domain $D$ and $\Gamma:[0,1) \rightarrow D$ a curve in $D$ landing at some point $p \in \partial_{\infty} D$. Then the curve $f^{-1} \circ \Gamma$ lands at some point $z_{0} \in \mathbb{T}:=\partial \mathbb{D}$. Moreover, if $\Gamma_{1}:[0,1) \rightarrow D$ is a curve in $D$ landing at another point $p_{1} \in \partial_{\infty} D \backslash\{p\}$, then the curve $f^{-1} \circ \Gamma_{1}$ lands at a point different from $z_{0}$.

Firstly we prove the following useful lemma.

Lemma 3.4. Suppose that $\varphi_{j}, j=1,2,3$, are holomorphic univalent self-mappings of $\mathbb{D}$, and $\varphi_{3}=\varphi_{2} \circ \varphi_{1}$. If any two of these three functions belong to $\mathcal{P}$, then so does the third one.

Proof. Let us assume that $\varphi_{2}$ and $\varphi_{3}$ belong to $\mathcal{P}$. Due to univalence of these functions, we have $\varphi_{1}=\varphi_{2}^{-1} \circ \varphi_{3}$. The function $\varphi_{3}$ has finite angular derivative at $z=1$. ¿From this one can conclude (see, e.g., [40, p. 80-81]) that $\varphi_{3}([0,1))$ is a $C^{1}$-smooth curve landing at the point $z=1$ and orthogonal to $\mathbb{T}:=\partial \mathbb{D}$ at this point. Since $\varphi_{2}$ is univalent, by Theorem $E$ there is a point $\xi \in \partial \mathbb{D}$ such that $\lim _{r \rightarrow 1-}\left(\varphi_{2}^{-1} \circ \varphi_{3}\right)(r)=\xi$. Again the existence of finite angular derivative of $\varphi_{2}$ at the point $z=1$ (see, e.g., [40, p. 8081]) implies that for any $\theta \in(0, \pi / 2)$ there exists $\vartheta \in(0, \pi / 2)$ and $r>0$ such that the domain $G_{r}(\theta):=\{z \in \mathbb{D}:|1-z|<r,|\arg (1-z)|<\theta\}$ is contained in the image $U:=\varphi_{2}(G)$ of $G=G_{1}(\vartheta)$. Moreover, for any $r>0$ and $\theta \in(0, \pi / 2)$ there exists $\rho \in(0,1)$ such that $\varphi_{3}([\rho, 1))$ is contained in $G_{r}(\theta)$. It follows that $\left(\varphi_{2}^{-1} \circ \varphi_{3}\right)([\rho, 1)) \subset G$. Hence $\xi=1$. From this we conclude that the function $\varphi_{1}=\varphi_{2}^{-1} \circ \varphi_{3}$ has at the point $z=1$ radial limit equal to 1. By the Lehto-Virtanen theorem (see, e.g., [39, Theorem 9.3 on 
p. 268]), this implies that $\varphi_{1}(1)=1$ in angular sense. According to Remark 3.2 the angular derivative $\varphi_{1}^{\prime}(1)$ exists, finite or infinite. Now one can use the chain rule for angular derivatives [16, Lemma 2] to conclude that the angular derivative $\varphi_{1}^{\prime}(1)$ actually equals 1 . This proves that $\varphi_{2} \in \mathcal{P}$.

Now assume that $\varphi_{1}$ and $\varphi_{2}$ belong to $\mathcal{P}$. Then the curve $\varphi_{2}([0,1))$ lands at $z=1$ nontangentially, i.e., $\varphi_{2}([0,1)) \subset G_{2}(\vartheta)$ for some $\vartheta<\pi / 2$. It follows that $\varphi_{3}(1)=1$ in angular sense. Therefore, we can apply the chain rule for angular derivatives to conclude that $\varphi_{3} \in \mathcal{P}$.

Finally, if $\varphi_{1}$ and $\varphi_{3}$ belong to $\mathcal{P}$, then the conclusion of the lemma follows immediately from [16, Lemma 2]. The proof is now finished.

Denote by $r_{\mathbb{H}}(\cdot, \cdot)$ the pseudo-hyperbolic distance in $\mathbb{H}$,

$$
r_{\mathbb{H}}(z, w)=\left|\frac{z-w}{z-\bar{w}}\right|, \quad z, w, \in \mathbb{H} .
$$

The hyperbolic distance $\rho_{\mathbb{H}}(z, w)$ between points $z, w \in \mathbb{H}$ then equals $\log [(1+r) /(1-r)]$, where $r:=r_{\mathbb{H}}(z, w)$.

Remark 3.5. We will frequently use a reformulation of the well-known growth estimate for the Carathéodory class (see, e.g., [39, p. 40, eq. (11)]). Namely, if $p$ is an analytic function in $\mathbb{H}$ with $\operatorname{Re} p(z) \geq 0$ for all $z \in \mathbb{H}$ and $\operatorname{Im} p\left(z_{0}\right)=0$ for some $z_{0} \in \mathbb{H}$, then

$$
|p(z)| \leq p\left(z_{0}\right) \frac{1+r_{\mathbb{H}}\left(z, z_{0}\right)}{1-r_{\mathbb{H}}\left(z, z_{0}\right)}
$$

for all $z \in \mathbb{H}$.

Now let us prove a very technical lemma.

Lemma 3.6. Let $b>0, z \in \mathbb{H}$. If $|z|>2 b$ and $|\arg z-\pi / 2| \leq \pi / 3$, then

$$
\frac{1+r_{\mathbb{H}}(z, i b)}{1-r_{\mathbb{H}}(z, i b)} \leq \frac{9|z|}{2 b}
$$

Proof. We start with the inequalities $\sqrt{1+x} \leq 1+x$ for $x \geq 0$, and $\sqrt{1+x} \geq 1+x$ for $x \in[-1,0]$. Using these inequalities we find that if $a>b$ and $\kappa \in[0,1]$, then

$$
\begin{aligned}
& \sqrt{a^{2}+b^{2}+2 \kappa a b} \geq a+b-\frac{2 a b}{a+b}(1-\kappa), \\
& \sqrt{a^{2}+b^{2}-2 \kappa a b} \leq a-b+\frac{2 a b}{a-b}(1-\kappa) .
\end{aligned}
$$

Applying the above inequalities to $a:=|z|$ and $\kappa:=\operatorname{Im} z /|z|$, we conclude that

$$
|z+i b| \geq|z|+b-\frac{2 b|z|}{|z|+b}(1-\kappa),
$$




$$
|z-i b| \leq|z|-b+\frac{2 b|z|}{|z|-b}(1-\kappa) .
$$

Since $|\arg z-\pi / 2| \leq \pi / 3$, we get that $\kappa \geq 1 / 2$. Therefore,

$$
|z+i b|-|z-i b| \geq 2 b\left(1-\frac{|z|^{2}}{|z|^{2}-b^{2}}(1-\kappa)\right) \geq \frac{2 b}{3} .
$$

Using (3.6), we get

$$
\frac{1+r_{\mathbb{H}}(z, i b)}{1-r_{\mathbb{H}}(z, i b)} \leq \frac{2|z+i b|}{|z+i b|-|z-i b|} \leq \frac{3}{b}|z+i b| \leq \frac{3}{b}|z|(1+b /|z|) \leq \frac{9|z|}{2 b},
$$

which finishes the proof.

To simplify the statement of the following two propositions, let us denote $\mathbb{N}_{0}:=\mathbb{N} \cup\{0\}$.

Proposition 3.7. Let $\left(\psi_{n}\right)_{n \in \mathbb{N}}$ be a sequence of functions from $\mathcal{P}$ and $\left(r_{j}\right)_{j \in \mathbb{N}_{0}}$ a sequence of numbers from $(-1,1)$ such that $\psi_{n}\left(r_{n}\right)=r_{0}$ for all $n \in \mathbb{N}$ and $\psi_{n}(\mathbb{D}) \subset \psi_{m}(\mathbb{D})$ whenever $n<m$. If

$$
\bigcup_{n \in \mathbb{N}} \psi_{n}(\mathbb{D})=\mathbb{D}
$$

then $r_{n} \rightarrow r_{0}$ and $\psi_{n} \rightarrow \mathrm{id}_{\mathbb{D}}$ as $n \rightarrow+\infty$.

Proof. To simplify and clarify the proof, it is better to present it in the framework of the upper half-plane. So assume that $\left(y_{n}\right)_{n \in \mathbb{N}}$ is a sequence of positive numbers and $\left(\psi_{n}\right)_{n \in \mathbb{N}}$ a sequence in $\mathfrak{P}$ such that $\psi_{n}(\mathbb{H}) \subset \psi_{m}(\mathbb{H})$ whenever $n<m$. Suppose that such that $\psi_{n}\left(i y_{n}\right)=i y_{0}$ for all $n \in \mathbb{N}$ and some $y_{0}>0$ not depending on $n$. Suppose also that

$$
\bigcup_{n \in \mathbb{N}} \psi_{n}(\mathbb{H})=\mathbb{H}
$$

We have to show that $y_{n} \rightarrow y_{0}$ and $\psi_{n} \rightarrow \mathrm{id}_{\mathbb{H}}$ as $n \rightarrow+\infty$.

Let us notice first that by Lemma 3.1, $y_{n} \leq y_{0}$ for all $n \in \mathbb{N}$. Further, suppose $m>n$. Then the inclusion $\psi_{n}(\mathbb{H}) \subset \psi_{m}(\mathbb{H})$ and Lemma 3.4 imply that $\psi_{n}=\psi_{m} \circ \omega_{n, m}$ for some $\omega_{n, m} \in \mathfrak{P}$, with $\omega_{n, m}\left(i y_{n}\right)=i y_{m}$. Again by Lemma 3.1, we have $y_{m} \geq y_{n}$. It follows that the sequence $\left(y_{n}\right)$ converges and let us denote the limit by $y_{\infty}$.

Given $f \in \operatorname{Hol}(\mathbb{H}, \mathbb{C})$, denote

$$
\begin{aligned}
I_{f}(z):=\left|\frac{f(z)}{z}-1\right|, z \in \mathbb{H}, & I_{f}(A):=\sup _{z \in A} I_{f}(z), A \subset \mathbb{H}, \\
J_{f}(z):=\frac{\operatorname{lm}(f(z)-z)}{|z|}, z \in \mathbb{H}, & J_{f}(A):=\sup _{z \in A} J_{f}(z), A \subset \mathbb{H} .
\end{aligned}
$$

Note that $0 \leq J_{f}(z) \leq I_{f}(z)$ for all $z \in \mathbb{H}$. 
We claim that for all $\varepsilon>0$ there exist a natural number $n$ and a positive number $R$ such that

$$
J_{\psi_{m}}(i y)<\varepsilon
$$

for all $m \geq n$ all all $y>R$.

Let us now prove this claim. Fix $\varepsilon \in(0,1 / 2)$. Let

$$
A:=\left\{z \in \mathbb{H}:|z| \geq 2 y_{\infty},|\arg z-\pi / 2| \leq \pi / 3\right\} .
$$

If $m \geq n$, using Remark 3.5, we can show that for any $z \in A$,

$$
I_{\omega_{n, m}}(z)=\left|\frac{\omega_{n, m}(z)-z}{z}\right| \leq \frac{1+r_{\mathbb{H}}\left(z, i y_{n}\right)}{1-r_{\mathbb{H}}\left(z, i y_{n}\right)} \cdot \frac{y_{m}-y_{n}}{|z|} .
$$

Applying now Lemma 3.6 for $b:=y_{n}$ we see that

$$
I_{\omega_{n, m}}(A) \leq \frac{9}{2 y_{n}}\left(y_{m}-y_{n}\right) \leq \frac{9}{2}\left(\frac{y_{\infty}}{y_{n}}-1\right) .
$$

Take $n_{0} \in \mathbb{N}$ such that $\frac{9}{2}\left(\frac{y_{\infty}}{y_{n_{0}}}-1\right)<\varepsilon$. For each $y \geq 3 y_{\infty}$, write

$$
A(y):=\{z \in A:|z| \geq 2 y / 3\} .
$$

Take $n \geq n_{0}$. Let us analyze how $\omega_{n, m}$ maps the boundary of $A(y)$. On one hand, if $z \in A$ with $|z|=2 y / 3$, then by (3.11), $\left|\omega_{n, m}(z)-z\right| \leq 2 y \varepsilon / 3$. On the other hand, if $z \in A$ and $\arg z=\pi / 6$, then using again (3.11) we can deduce $\left|\arg \omega_{n, m}(z)-\pi / 6\right| \leq \arcsin \varepsilon$. Similarly, if $z \in A$ and $\arg z=5 \pi / 6$, then $\left|\arg \omega_{n, m}(z)-5 \pi / 6\right| \leq \arcsin \varepsilon$. This behavior of $\omega_{n, m}$ on the boundary of $A(y)$ clearly implies that the half-line $[2 y(1+\varepsilon) / 3,+\infty) i$ is contained in the set $\omega_{n, m}(A(y))$. In particular, iy $\in \omega_{n, m}(A(y))$.

Since $\psi_{n}=\psi_{m} \circ \omega_{n, m}$, given $z \in A$ we have

$$
\begin{array}{r}
J_{\psi_{n}}(z)=\frac{\operatorname{Im}\left(\psi_{m}\left(\omega_{n, m}(z)\right)-z\right)}{|z|}=\frac{\operatorname{Im}\left(\psi_{m}\left(\omega_{n, m}(z)\right)-\omega_{n, m}(z)\right)}{|z|}+\frac{\operatorname{Im}\left(\omega_{n, m}(z)-z\right)}{|z|} \\
=J_{\psi_{m}}\left(\omega_{n, m}(z)\right) \frac{\left|\omega_{n, m}(z)\right|}{|z|}+J_{\omega_{n, m}}(z) \geq J_{\psi_{m}}\left(\omega_{n, m}(z)\right) \frac{\left|\omega_{n, m}(z)\right|}{|z|} .
\end{array}
$$

It follows that for any $y \geq 3 y_{\infty}$ and $n \geq n_{0}$,

$$
J_{\psi_{m}}(i y) \leq \frac{J_{\psi_{n}}(A(y))}{1-I_{\omega_{n, m}}(A)} \leq \frac{\varepsilon}{1-\varepsilon} \leq 2 \varepsilon .
$$

This finishes the proof of the claim.

Now let us consider the function $\phi_{n}(z):=\psi_{n}\left(y_{n} L_{\alpha_{n}}\left(z / y_{0}\right)\right)$, where $L_{\alpha}$ is a non-Euclidian rotation, $L_{\alpha}(z)=H\left(\alpha H^{-1}(z)\right), H(\zeta):=i(1+\zeta) /(1-\zeta)$, and $\alpha_{n}:=\left|\psi_{n}^{\prime}\left(i y_{n}\right)\right| / \psi_{n}^{\prime}\left(i y_{n}\right)$. Since $\phi_{n}\left(i y_{0}\right)=i y_{0}$ and $\phi_{n}^{\prime}\left(i y_{0}\right)>0$, by the Carathéodory kernel theorem (see, e.g., [21, Theorem 1 on p. 55]) it follows from (3.8) that $\phi_{n} \rightarrow \mathrm{id}_{\mathbb{H}}$ as $n \rightarrow+\infty$. Moreover, passing to a subsequence, we can assume that $\left(\alpha_{n}\right)$ converges to some $\alpha_{0}$. Consequently, 
$\psi_{n}(z) \rightarrow y_{0} L_{\alpha_{0}}^{-1}\left(z / y_{\infty}\right)$ as $n \rightarrow+\infty$. At the same time, according to (3.9) composed with Lemma 3.1, the limit function of $\left(\psi_{n}\right)$ should have a parabolic boundary fixed point at $\tau=\infty$, i. e. $\alpha_{0}=1$ and $y_{\infty}=y_{0}$. This completes the proof.

Proposition 3.8. Let $\left(\psi_{n}\right)_{n \in \mathbb{N}}$ be a sequence of functions from $\mathcal{P}$ and $\left\{r_{j}\right\}_{j \in \mathbb{N}_{0}}$ a sequence of numbers from $(-1,1)$ such that $r_{n}:=\psi_{n}\left(r_{0}\right)$ for $n \in \mathbb{N}$. Suppose that there exists a sequence of conformal self-mappings $\left(\phi_{j}\right)_{j \in \mathbb{N}_{0}}$ of $\mathbb{D}$ normalized by $\phi_{j}(0)=0, \phi_{j}^{\prime}(0)>0$, $j \in \mathbb{N}_{0}$, and a sequence $\left(L_{j}\right)_{j \in \mathbb{N}_{0}} \subset \operatorname{Möb}(\mathbb{D})$ such that $\phi_{n} \circ L_{n} \circ \psi_{n}=\phi_{0} \circ L_{0}$ for all $n \in \mathbb{N}$. If $\phi_{m}(\mathbb{D}) \subset \phi_{n}(\mathbb{D})=: G_{n}$ whenever $m \geq n>0$ and if $G_{0}:=\phi_{0}(\mathbb{D})$ is a connected component of the intersection $\cap_{n \in \mathbb{N}} G_{n}$, then $r_{n} \rightarrow r_{0}$ and $\psi_{n} \rightarrow \mathrm{id}_{\mathbb{D}}$ as $n \rightarrow+\infty$.

Proof. We again present the proof in the framework of self-maps of the upper half-plane. So, let $\left(\psi_{n}\right)_{n \in \mathbb{N}} \subset \mathfrak{P}$ and $\psi_{n}\left(i y_{0}\right)=i y_{n}, n \in \mathbb{N}$, for some sequence $\left(y_{j}\right)_{j \in \mathbb{N}_{0}}$ of positive numbers. Assume that there exists a sequence of conformal mappings $\left(\phi_{j}\right)_{j \in \mathbb{N}_{0}}$ of $\mathbb{H}$ into itself normalized by $\phi_{j}(i)=i, \phi_{j}^{\prime}(i)>0, j \in \mathbb{N}_{0}$, and a sequence $\left(L_{j}\right)_{j \in \mathbb{N}_{0}} \subset \operatorname{Möb}(\mathbb{H})$ such that:

(1) $\phi_{n} \circ L_{n} \circ \psi_{n}=\phi_{0} \circ L_{0}$ for all $n \in \mathbb{N}$,

(2) $\phi_{m}(\mathbb{H}) \subset \phi_{n}(\mathbb{H})=: G_{n}$ whenever $m \geq n>0$, and

(3) $G_{0}:=\phi_{0}(\mathbb{H})$ is a connected component of the interior of $\bigcap_{n \in \mathbb{N}} G_{n}$.

We have to prove that $y_{n} \rightarrow y_{0}$ and $\psi_{n} \rightarrow \mathrm{id}_{\mathbb{H}}$ as $n \rightarrow+\infty$.

According to the Carathéodory kernel theorem (see, e.g., [21, Theorem 1 on p. 55]), $\phi_{n}^{-1} \rightarrow \phi_{0}^{-1}$ uniformly on compact subsets of $G_{0}$ as $n \rightarrow+\infty$. By Lemma 3.4 we have

$$
L_{n} \circ \psi_{n} \rightarrow L_{0}, \quad(n \rightarrow+\infty) .
$$

Since $\left(\psi_{n}\right)$ is a normal family, each subsequence has a further subsequence that converges either to a holomorphic function mapping $\mathbb{H}$ into itself or to a constant in $\mathbb{R} \cup\{\infty\}$. Let us consider one of such limits, which we denote by $\psi$. It suffices to prove that $\psi=\mathrm{id}_{\mathbb{H}}$.

Let $m \geq n>0$. Observe that $\phi_{m}=\phi_{n} \circ \tilde{\omega}_{n, m}$ for some holomorphic self-mapping $\tilde{\omega}_{n, m}$ of $\mathbb{H}$. It follows that

$$
\psi_{n}=\omega_{n, m} \circ \psi_{m}, \quad \text { with some } \omega_{n, m} \in \mathfrak{P} .
$$

In particular, $i y_{n}=\omega_{n, m}\left(i y_{m}\right)$. Then, by Remark 3.3, we conclude that $y_{n} \geq y_{m}$ for $m \geq n>0$. Again by Remark 3.3, $y_{n} \geq y_{0}>0$. It follows that $\left(y_{n}\right)$ converges to some $y_{\infty} \in(0,+\infty)$. Consequently, $\psi\left(i y_{0}\right)=i y_{\infty} \in \mathbb{H}$. Therefore, (3.13) implies that $\psi \in$ $\operatorname{Möb}(\mathbb{H})$. Bearing in mind that $\operatorname{Re} \psi\left(i y_{0}\right)=0$, we see that to prove $\psi=\mathrm{id}_{\mathbb{H}}$ it is sufficient to show that $\psi \in \mathfrak{P}$. In its turn, the proof of this statement can be reduced with the help of Lemma 3.1 to proving that

$$
(\forall \varepsilon>0)(\exists R>0)(\forall m \in \mathbb{N}, y>R) J_{\psi_{m}}(i y)<\varepsilon,
$$

where

$$
J_{f}(z):=\frac{\operatorname{lm}(f(z)-z)}{|z|}
$$


Taking into account (3.14), we get

$$
\begin{aligned}
J_{\psi_{1}}(i y)=\frac{\operatorname{Im}\left(\omega_{1, m}\left(\psi_{m}(i y)\right)-i y\right)}{y}=\frac{\operatorname{Im}\left(\omega_{1, m}\left(\psi_{m}(i y)\right)-\psi_{m}(i y)\right)}{y}+ \\
\quad \frac{\operatorname{Im}\left(\psi_{m}(i y)-i y\right)}{y}=J_{\omega_{1, m}}\left(\psi_{m}(i y)\right) \cdot \frac{\left|\psi_{m}(i y)\right|}{y}+J_{\psi_{m}}(i y) \geq J_{\psi_{m}}(i y),
\end{aligned}
$$

because by Remark $3.3, J_{f}(z) \geq 0$ for all $f \in \mathfrak{P}$ and $z \in \mathbb{H}$. In view of Lemma 3.1 applied to $\psi_{1}$, this inequality implies (3.15) and thus completes the proof.

\section{L-ADMISSIBLE FAMILIES AND CHORDAL EVOLUTION FAMILIES}

4.1. Chordally admissible families. Let us denote by $\mathrm{P}(D)$ the Carathéodory boundary of a domain $D$, i. e., the set of all prime ends of $D$. For a Jordan domain $D$, the Carathéodory boundary $\mathrm{P}(D)$ can be identified with the topological boundary $\partial D$.

Let $F$ be a conformal mapping of a simply connected domain $D$. By $F^{p . e}$. we will denote the bijection between $\mathrm{P}(D)$ and $\mathrm{P}(F(D))$ induced by $F$.

Definition 4.1. Let $G$ be a simply connected subdomain of $\mathbb{D}, \psi: \mathbb{D} \rightarrow G$ a conformal mapping, $P \in \mathrm{P}(G)$, and $\zeta_{0}:=\left(\psi^{\text {p.e. }}\right)^{-1}(P)$. We will say that $G$ is embedded in $\mathbb{D}$ conformally at the prime end $P$ if $\zeta_{0}$ is a regular contact point of the function $\psi$, i. e., the following conditions hold:

(i) $\exists \angle \lim _{\zeta \rightarrow \zeta_{0}} \psi(\zeta):=z_{0} \in \mathbb{T}$;

(ii) $\psi^{\prime}\left(\zeta_{0}\right):=\angle \lim _{\zeta \rightarrow \zeta_{0}} \frac{\psi(\zeta)-z_{0}}{\zeta-\zeta_{0}} \neq \infty$.

Definition 4.1 can be extended to the case of two arbitrary hyperbolic simply connected domains $\Omega_{1} \subset \Omega_{2}$ :

Definition 4.2. Let $\Omega_{1}$ and $\Omega_{2}$ be two hyperbolic simply connected domains in the complex plain with $\Omega_{1} \subset \Omega_{2}$. The domain $\Omega_{1}$ is said to be embedded in the domain $\Omega_{2}$ conformally at a prime end $P_{1} \in \mathrm{P}\left(\Omega_{1}\right)$ if there is a conformal mapping $\phi: \Omega_{2} \rightarrow \mathbb{D}$ such that the domain $G:=\phi\left(\Omega_{1}\right)$ is embedded in $\mathbb{D}$ conformally at the prime end $P:=$ $\left(\left.\phi\right|_{\Omega_{1}}\right)^{\text {p.e. }}\left(P_{1}\right) \in \mathrm{P}(G)$.

Remark 4.3. It is clear that Definition 4.1 above does not depend on particular normalization of the conformal mappings $\psi$ and $\phi$. More precisely, if $\psi_{1}$ and $\psi_{2}$ are two conformal mappings of $\mathbb{D}$ onto $G$ and $\zeta_{j}:=\left(\psi_{j}^{p . e .}\right)^{-1}(P), j=1,2$, then $\psi:=\psi_{1}$ with $\zeta_{0}:=\zeta_{1}$ satisfies conditions (i) and (ii) in this definition if and only if they are fulfilled by $\psi:=\psi_{2}$ with $\zeta_{0}:=\zeta_{2}$. Similar remark concerns the conformal mapping $\phi$ in Definition 4.2 .

Definition 4.4. By $\mathcal{C}$ we denote the class of all univalent functions $\varphi \in \operatorname{Hol}(\mathbb{D}, \mathbb{D})$ such that

$$
\angle \lim _{z \rightarrow 1} \varphi(z)=1, \quad \varphi^{\prime}(1):=\angle \lim _{z \rightarrow 1} \frac{\varphi(z)-1}{z-1}<\infty
$$


and let

$$
\mathcal{C}_{0}:=\left\{\varphi \in \mathcal{C}: \varphi^{\prime}(1) \leq 1\right\}
$$

Lemma 4.5. Let $\Omega_{1}$ and $\Omega_{2}$ be two hyperbolic simply connected domains in the complex plain with $\Omega_{1} \subset \Omega_{2}$. There is a prime end $P_{1} \in \mathrm{P}\left(\Omega_{1}\right)$ such that the domain $\Omega_{1}$ is embedded in the domain $\Omega_{2}$ conformally at $P_{1}$ if and only if there exist two univalent functions $F_{j}$, $j=1,2$, in the unit disk $\mathbb{D}$ such that

$$
F_{j}(\mathbb{D})=\Omega_{j}, j=1,2 \text {, and } F_{2}^{-1} \circ F_{1} \in \mathcal{C} .
$$

Proof. Suppose first that there exists $P_{1} \in \mathrm{P}\left(\Omega_{1}\right)$ such that $\Omega_{1}$ is embedded in $\Omega_{2}$ conformally at $P_{1}$. Take any $w_{0} \in \Omega_{1}$ and choose $F_{1}$ to be the conformal mapping of $\mathbb{D}$ onto $\Omega_{1}$ normalized by $F_{1}(0)=w_{0}$ and $F_{1}^{p . e .}(1)=P_{1}$. Let $\phi$ be any conformal mapping of $\Omega_{2}$ onto $\mathbb{D}$ subject to the condition $\phi\left(w_{0}\right)=0$. The function $\psi:=\phi \circ F_{1}$ maps $\mathbb{D}$ conformally into itself and satisfies conditions (i) and (ii) in Definition 4.1 with

$$
\zeta_{0}=\left(\psi^{\text {p.e. }}\right)^{-1}\left(\left(\left.\phi\right|_{\Omega_{1}}\right)^{\text {p.e. }}\left(P_{1}\right)\right)=\left(F_{1}^{-1} \circ\left(\left.\phi\right|_{\Omega_{1}}\right)^{-1}\right)^{\text {p.e. }}\left(\left(\left.\phi\right|_{\Omega_{1}}\right)^{\text {p.e. }}\left(P_{1}\right)\right)=\left(F_{1}^{\text {p.e. }}\right)^{-1}\left(P_{1}\right)=1 \text {. }
$$

It follows that $\left(1 / z_{0}\right) \phi \circ F_{1} \in \mathcal{C}$, where $z_{0} \in \mathbb{T}$ stands for the angular limit of $\psi$ at $\zeta_{0}$. Therefore, we conclude just by choosing $F_{2}(z):=\phi^{-1}\left(z_{0} z\right)$.

Conversely, let us now assume that there exist two univalent functions $F_{j}, j=1,2$, in the unit disk $\mathbb{D}$ satisfying (4.2). Let $\psi:=F_{2}^{-1} \circ F_{1}$. According to Definition 4.1 and Remark 4.3 the domain $G:=F_{2}^{-1}\left(\Omega_{1}\right)$ is embedded conformally in $\mathbb{D}$ at the prime end $P:=$ $\psi^{\text {p.e. }}(1)$. From this we conclude in a similar way, using Definition 4.2 with $\phi=F_{2}^{-1}$, that $\Omega_{1}$ is embedded conformally in $\Omega_{2}$ at the prime end $P_{1}:=F_{1}^{p . e .}(1)$. The proof is now completed.

Definition 4.6. An L-admissible family $\left(\Omega_{t}\right)$ is said to be chordally admissible if there exists a prime end $P_{0} \in \mathrm{P}\left(\Omega_{0}\right)$ such that for each $t \geq 0$ the domain $\Omega_{0}$ is embedded in the domain $\Omega_{t}$ conformally at the prime end $P_{0}$.

Clearly, Lemma 4.5 implies

Lemma 4.7. An L-admissible family $\left(\Omega_{t}\right)$ is chordally admissible if and only if there exist a family $\left(F_{t}\right)$ of univalent holomorphic functions in the unit disk $\mathbb{D}$ such that

$$
F_{t}(\mathbb{D})=\Omega_{t} \text { and } F_{t}^{-1} \circ F_{0} \in \mathcal{C} \quad \text { for all } t \geq 0 .
$$

Let us denote by $\Lambda^{d}$ the set of all locally absolutely continuous functions $\lambda:[0,+\infty) \rightarrow \mathbb{C}$ fulfilling the following conditions:

(i) $\lambda^{\prime} \in L_{\text {loc }}^{d}([0,+\infty), \mathbb{C})$;

(ii) $\lambda(0)=0$;

(iii) $\operatorname{Re} \lambda(t) \geq \operatorname{Re} \lambda(s)$ for any $s \geq 0$ and $t \geq s$.

Let $\Lambda_{R}^{d}$ stand for the subclass of all real-valued functions from $\Lambda^{d}$.

In [9, Theorem 7.1] it was proved that for any chordal or radial evolution family $\left(\varphi_{s, t}\right)$ with common Denjoy-Wolff point $\tau$ we have $\varphi_{s, t}^{\prime}(\tau)=\exp (\lambda(s)-\lambda(t))$ for all $s \geq 0$, 
$t \geq s$ and some $\lambda \in \Lambda^{d}$. Moreover, if $\left(\varphi_{s, t}\right)$ is chordal, i.e. $\tau \in \mathbb{T}:=\partial \mathbb{D}$, then $\lambda \in \Lambda_{R}^{d}$. Now let us formulate the Main Theorem.

Theorem 4.8. Let $\left(\Omega_{t}\right)$ be an L-admissible family $\left(\Omega_{t}\right)$ of order $d \in[1,+\infty]$ and $\tau \in \mathbb{T}:=\partial \mathbb{D}$. Then:

1. The following statements are equivalent:

(a) For all $\lambda \in \Lambda_{R}^{d}$ there exists an evolution family $\left(\varphi_{s, t}\right)$ of order $d$ and a Loewner chain $\left(f_{t}\right)$ associated with $\left(\varphi_{s, t}\right)$ such that:

(A.1) $\varphi_{s, t}(\tau)=\tau$ and $\varphi_{s, t}^{\prime}(\tau)=\exp (\lambda(s)-\lambda(t))$ for all $s \geq 0$ and $t \geq s$;

(A.2) $\left\{f_{t}(\mathbb{D}): t \geq 0\right\}=\left\{\Omega_{t}: t \geq 0\right\}$.

(b) For at least one $\lambda \in \Lambda_{R}^{d}$ there exists an evolution family $\left(\varphi_{s, t}\right)$ of order $d$ and a Loewner chain $\left(f_{t}\right)$ associated with $\left(\varphi_{s, t}\right)$ such that the above conditions (A.1) and (A.2) are fulfilled.

(c) The family $\left(\Omega_{t}\right)$ is chordally admissible.

2. The following statements are equivalent:

(d) For all $\lambda \in \Lambda_{R}^{d}$ there exists an evolution family $\left(\varphi_{s, t}\right)$ of order $d$ and a Loewner chain $\left(f_{t}\right)$ associated with $\left(\varphi_{s, t}\right)$ such that:

(B.1) $\varphi_{s, t}(\tau)=\tau$ and $\varphi_{s, t}^{\prime}(\tau)=\exp (\lambda(s)-\lambda(t))$ for all $s \geq 0$ and $t \geq s$;

(B.2) $f_{t}(\mathbb{D})=\Omega_{t}(\mathbb{D})$ for all $t \geq 0$.

(e) For at least one $\lambda \in \Lambda_{R}^{d}$ there exists an evolution family $\left(\varphi_{s, t}\right)$ of order $d$ and a Loewner chain $\left(f_{t}\right)$ associated with $\left(\varphi_{s, t}\right)$ such that the above conditions (B.1) and (B.2) are fulfilled.

\subsection{Proof of the Main Theorem.}

4.2.1. Auxiliary statements. First we prove an analogue of [17, Proposition 2.10] for functions with common parabolic fixed point on the boundary. Let us recall that by $A C^{d}(X, Y), X \subset \mathbb{R}, d \in[1,+\infty]$, we denote the class of all locally absolutely continuous functions $f: X \rightarrow Y$ such that the derivative $f^{\prime}$ belongs to $L_{\text {loc }}^{d}(X)$.

Proposition 4.9. Let $\left(\varphi_{s, t}\right)_{0 \leq s \leq t<+\infty}$ be a family of functions from class $\mathcal{P}$ and $d \in[1,+\infty]$. Suppose that $\left(\varphi_{s, t}\right)$ satisfies conditions EF1 and EF2 in Definition 1.1. If the function $t \mapsto a(t):=\varphi_{0, t}(0)$ belongs to $A C_{\text {loc }}^{d}([0,+\infty), \mathbb{R})$, then $\left(\varphi_{s, t}\right)$ is an evolution family of order $d$.

Proof. It is more convenient to work with the family $\left(\psi_{s, t}\right)$ defined by $\psi_{s, t}=H \circ \varphi_{s, t} \circ H^{-1}$, where $H$ stands for the Cayley map (3.1). Then for any $s \geq 0$ and $t \geq s$, we have $\psi_{s, t}(z)=z+i p_{s, t}(z)$, where $p_{s, t}$ is some analytic function in $\mathbb{H}$ having non-negative real part. The equality $\varphi_{0, t}(0)=a(t)$ implies that $p_{s, t}(i b(s))=b(t)-b(s), 0 \leq s \leq t$, where

$$
b(t):=(1 / i) H(a(t))=\frac{1+a(t)}{1-a(t)} .
$$


The key idea is to take advantage of inequality (3.4) in Remark 3.5. Apply this inequality for $p:=p_{u, t}$ with $z_{0}:=i b(u)$ and $z:=\psi_{s, u}(\zeta), \zeta \in \mathbb{H}$, to obtain

$$
\left|\psi_{s, t}(\zeta)-\psi_{s, u}(\zeta)\right|=\left|\psi_{u, t}(z)-z\right| \leq \frac{1+r}{1-r}|b(t)-b(u)|, \quad r:=r_{\mathbb{H}}\left(i b(u), \psi_{s, u}(\zeta)\right) .
$$

Since any analytic self-map of $\mathbb{H}$ is a contraction for the hyperbolic metric, we have

$$
r_{\mathbb{H}}\left(i b(u), \psi_{s, u}(\zeta)\right)=r_{\mathbb{H}}\left(\psi_{s, u}(i b(s)), \psi_{s, u}(\zeta)\right) \leq r_{\mathbb{H}}(i b(s), \zeta) .
$$

Combining inequalities (4.4) and (4.5) and taking into account that $b$ is continuous, we conclude that for any $\zeta \in \mathbb{H}$ and any $T \in[0,+\infty)$ there exists a constant $C=C(\zeta, T)>0$ such that $\left|\psi_{s, t}(\zeta)-\psi_{s, u}(\zeta)\right| \leq C|b(t)-b(u)|$ whenever $0 \leq s \leq u \leq t \leq T$. Bearing in mind that $|w-z| \leq|H(w)-H(z)|$ for any $z, w \in \mathbb{D}$, we see that this inequality proves the proposition.

Let us formulate the most difficult part to prove in Theorem 4.8,

Theorem 4.10. Let $\tau \in \mathbb{T}:=\partial \mathbb{D}$. For any chordally admissible family $\left(\Omega_{t}\right)$ there exists an evolution family $\left(\varphi_{s, t}\right)$ of order $+\infty$ and a Loewner chain $\left(f_{t}\right)$ associated with $\left(\varphi_{s, t}\right)$ such that $\left\{f_{t}(\mathbb{D}): t \geq 0\right\}=\left\{\Omega_{t}: t \geq 0\right\}$ and $\varphi_{s, t}(\tau)=\tau, \varphi_{s, t}^{\prime}(\tau)=1$ for each $s \geq 0$ and $t \geq s$.

Proof. Without loss of generality one can assume that $\tau=1$. We divide the proof into the four following steps.

Step 1. There exists a family $\left(h_{t}\right)_{t \geq 0} \subset \operatorname{Hol}(\mathbb{D}, \mathbb{C})$ that satisfies the following conditions:

(i) $h_{t}(\mathbb{D})=\Omega_{t}$ for all $t \geq 0$;

(ii) the mappings $\phi_{s, t}:=h_{t}^{-1} \circ h_{s}, 0 \leq s \leq t$, belong to the class $\mathcal{P}$,

(iii) $\phi_{0, t}(0) \in(-1,1)$ for all $t \geq 0$.

This part is easy. By hypothesis, $\left(\Omega_{t}\right)$ is a chordally admissible family. Hence by Lemma 4.7, there exists a family $\left(F_{t}\right)$ of univalent functions in the unit disk $\mathbb{D}$ satisfying (4.3). Write $\Phi_{t}=F_{t}^{-1} \circ F_{0}$.

Now let us make use of the following obvious assertion: for any function $\varphi \in \mathcal{C}$ there exists $m[\varphi] \in \operatorname{Möb}(\mathbb{D})$ such that $m[\varphi] \circ f \in \mathcal{P}$ and $\operatorname{Im} m[\varphi](\varphi(0))=0$. This Möbius transformation can be constructed as a composition of three transformations:

$$
z \mapsto \frac{z-\varphi(0)}{1-\overline{\varphi(0)} z}, z \mapsto e^{i \theta} z, \text { and } z \mapsto \frac{z-a}{1-a z},
$$

for appropriately chosen $\theta \in \mathbb{R}$ and $a \in(-1,1)$. Setting $\varphi:=\Phi_{t}$ and taking into account that $\Phi_{0}=\mathrm{id}_{\mathbb{D}}$, we conclude that the functions $h_{t}:=F_{t} \circ\left(m\left[\Phi_{t}\right]\right)^{-1}, t \geq 0$, form a family satisfying condition (i), (ii) and (iii), for all $t \geq 0$.

In order to establish (ii) for all $s \in[0, t]$ it suffices to notice that $\phi_{0, t}=\phi_{s, t} \circ \phi_{0, s}$, and apply Lemma 3.4 to make sure that $\phi_{s, t}$ is also in $\mathcal{P}$.

Step 2. The mapping $\mathcal{Q}: t \mapsto \phi_{0, t}=h_{t}^{-1} \circ h_{0}$ from $[0,+\infty)$ into $\mathrm{Hol}(\mathbb{D}, \mathbb{D})$ is continuous. 
Denote $x(t):=\phi_{0, t}(0) \in(-1,1)$. Let $t_{n} \in[0,+\infty)$ be such that the sequence $\left(t_{n}\right)$ converges to a point $t_{0}$. The assertion of Step 2 will be proved if we show that $\phi_{0, t_{n}}$ converges to $\phi_{0, t_{0}}$, as $n \rightarrow+\infty$, uniformly on compact subsets of the unit disk. We can make even further simplification without loss of generality. Namely, we can assume that $\left(t_{n}\right)$ is either increasing or decreasing. Let us start with the first case.

Step 2a. If $t_{n}$ is increasing, then $\phi_{0, t_{n}} \rightarrow \phi_{0, t_{0}}$ as $n \rightarrow+\infty$.

For $n \in \mathbb{N}_{0}$ we denote $\psi_{n}:=\phi_{t_{n}, t_{0}}$ and $r_{n}:=x\left(t_{n}\right)$. Note that $\psi_{n} \in \mathcal{P}$. Further, observe that $\psi_{n}(\mathbb{H})=h_{t_{0}}^{-1}\left(h_{t_{n}}(\mathbb{D})\right)$. By this reason, $\psi_{n}(\mathbb{H}) \subset \psi_{m}(\mathbb{H})$ whenever $0 \neq n<m$. By assertion (ii) in Remark 1.7 we have $\cup_{n \in \mathbb{N}} \psi_{n}(\mathbb{H})=\mathbb{H}$. Summarizing the above argument we see that $\left(\psi_{n}\right)$ satisfies the hypothesis of Proposition 3.7. As a result we conclude that $\psi_{n} \rightarrow \psi_{0}=\mathrm{id}_{\mathbb{H}}$ as $n \rightarrow+\infty$. Since $\psi_{n} \circ \phi_{0, t_{n}}=\phi_{0, t_{0}}$ is independent of $n$, this implies that $\phi_{0, t_{n}}$ converges to $\phi_{0, t_{0}}$.

Therefore, the proof of Step 2a is now finished and we proceed with considering the case of decreasing sequence $\left(t_{n}\right)$.

Step 2b. If $t_{n}$ is decreasing, then $\phi_{0, t_{n}} \rightarrow \phi_{0, t_{0}}$ as $n \rightarrow+\infty$.

As in the previous case, we denote $r_{n}:=x\left(t_{n}\right), n \in \mathbb{N}_{0}$. Set $\psi_{n}:=\phi_{t_{0}, t_{n}} \in \mathcal{P}$. Then we have $\psi_{n}\left(r_{0}\right)=r_{n}$ for all $n \in \mathbb{N}$. Set $T:=t_{1}$. Consider mappings $\phi_{t_{n}, T}, n \in \mathbb{N}_{0}$, and let $L_{n}$ be the unique function in $\operatorname{Möb}(\mathbb{D})$ such that the functions $\phi_{n}:=\phi_{t_{n}, T} \circ L_{n}^{-1}, n \in \mathbb{N}_{0}$, satisfy the normalization $\phi_{n}(0)=0$ and $\phi_{n}^{\prime}(0)>0$, required in Proposition 3.8. Then we have

$$
\phi_{n} \circ L_{n} \circ \psi_{n}=\phi_{t_{n}, T} \circ \phi_{t_{0}, t_{n}}=\phi_{t_{0}, T}=\phi_{0} \circ L_{0} .
$$

Furthermore, the domains $G_{n}:=\phi_{n}(\mathbb{D})=\phi_{t_{n}, T}(\mathbb{D})=h_{T}^{-1}\left(h_{t_{n}}(\mathbb{D})\right)$ form, starting from $n=1$, a contracting sequence of domains, with $G_{0}$ being, according to assertion (i) in Remark 1.7, a connected component of the interior of $\cup_{n \in \mathbb{N}} G_{n}$.

Hence, we can apply Proposition 3.8 to conclude that $\psi_{n} \rightarrow \mathrm{id}_{H}$ as $n \rightarrow+\infty$. As a consequence, $\phi_{0, t_{n}}$ converges to $\phi_{0, t_{0}}$, which is what we had to show in order to complete the proof of Step $2 \mathrm{~b}$.

Step 3. Let $x(t):=\phi_{0, t}(0)$. There exists a non-decreasing function $\theta:[0,+\infty) \rightarrow[0,+\infty)$ with $\theta(0)=0$ such that $t \mapsto x(\theta(t))$ is a smooth function on $[0,+\infty)$ and

$$
\left\{h_{\theta(t)}: t \geq 0\right\}=\left\{h_{t}: t \geq 0\right\} .
$$

Let us first of all mention that $x(0)=0$ and that $x(t)=\phi_{s, t}(x(s))$ for any $s \geq 0$ and $t \geq s$. Passing this equation to the upper half-plane and using that the function $x$ is real valued, from Remark 3.3 it follows that the function $t \mapsto x(t), t \in[0,+\infty)$, is non-decreasing. Furthermore, by Step 2 this function is continuous.

In particular, it follows that $J:=x([0,+\infty))$ is an interval of the form $[0, a)$ or $[0, a]$ for some $a \in(0,1]$. Take a function $\chi:[0,+\infty) \rightarrow J$ smooth and non-decreasing. Now for each $t \geq 0$ let us set

$$
\theta(t):=\inf \{\theta \geq 0: x(\theta)=\chi(t)\}
$$


Since $x(0)=0$, immediately we deduce $\theta(0)=0$. Since the functions $\theta \mapsto x(\theta)$ and $t \mapsto \chi(t)$ have the same range on $[0,+\infty)$, the function $t \mapsto \theta(t)$ is well defined on $[0,+\infty)$. Furthermore, since $\theta \mapsto x(\theta)$ is continuous it follows that $x(\theta(t))=\chi(t)$ for all $t \geq 0$, which means that $t \mapsto x(\theta(t))$ is smooth. Finally, $t \mapsto \theta(t)$ is non-decreasing because both functions $\theta \mapsto x(\theta)$ and $t \mapsto \chi(t)$ are non-decreasing.

It remains to show that (4.6) holds. To this end denote $\Theta:=\theta([0,+\infty))$ and let us again apply Remark 3.3 and equality $x(t)=\phi_{s, t}(x(s))$ to conclude that $x(s)=x(t)$ holds for some $t, s \geq 0$ if and only if $\phi_{s, t}=\mathrm{id}_{\mathbb{D}}$, which is in its turn equivalent to $h_{t}=h_{s}$. It follows that for any two sets $A_{1}, A_{2} \in[0,+\infty)$ the ranges of $t \mapsto h_{t}$ on $A_{1}$ and on $A_{2}$ coincide if and only if the same is true for $t \mapsto x(t)$. By construction, the range of $t \mapsto x(t)$ on $\Theta$ is the same as on the whole semiaxis $[0,+\infty)$. This implies (4.6) and finishes the proof of Step 3.

Step 4. The family $\left(\varphi_{s, t}\right)$ defined by $\varphi_{s, t}=\phi_{\theta(s), \theta(t)}, 0 \leq s \leq t$, is an evolution family of order $+\infty$ and the family $\left(f_{t}\right)$ defined by $f_{t}:=h_{\theta(t)}$ is a Loewner chain associated with the evolution family $\left(\varphi_{s, t}\right)$.

Clearly, the family $\left(\varphi_{s, t}\right)$ satisfies conditions EF1 and EF2 in Definition 1.1. Moreover, by the construction, the function $t \mapsto \varphi_{0, t}(0)$ is real-valued and smooth. Hence, by Proposition 4.9, $\left(\varphi_{s, t}\right)$ is an evolution family of order $d=+\infty$. Note that $f_{t} \circ \varphi_{s, t}=f_{s}$ for all $s \geq 0$ and $t \geq s$. Then by [17, Lemma 3.2], $\left(f_{t}\right)$ is a Loewner chain of order $d=\infty$.

The proof of Theorem 4.10 is now completed.

The following statement is a direct consequence of [17, Lemma 2.8].

Lemma 4.11. Let $\tau \in \mathbb{T}$ and $\lambda \in \Lambda_{R}^{d}$. Let $\left(\psi_{s, t}\right)$ be a family of holomorphic self-maps of $\mathbb{D}$, and

$$
\varphi_{s, t}:=h_{t} \circ \psi_{s, t} \circ h_{s}^{-1}, \quad t \geq s \geq 0, \quad h_{t}(z):=\tau \frac{z-\tau a(t)}{\tau-a(t) z}, a(t):=\frac{e^{\lambda(t)}-1}{e^{\lambda(t)}+1} .
$$

Then the following two statements are equivalent:

(i) the family $\left(\varphi_{s, t}\right)$ is an evolution family of order $d$ with $\varphi_{s, t}(\tau)=\tau$ and $\varphi_{s, t}^{\prime}(\tau)=$ $\exp (\lambda(t)-\lambda(s))$ for all $s \geq 0$ and $t \geq s$;

(ii) the family $\left(\psi_{s, t}\right)$ is an evolution family of order $d$ with $\psi_{s, t}(\tau)=\tau$ and $\psi_{s, t}^{\prime}(\tau)=1$ for all $s \geq 0$ and $t \geq s$.

Now we are ready to prove Theorem 4.8.

4.2.2. Proof of Statement 1 of Theorem 4.8. Let us use Lemma 4.11 in order to deduce Statement 1 of Theorem 4.8 from Theorem 4.10. Clearly, in view of Theorem 4.10, (c) implies (b). Let us show that (c) also implies (a). To this end take an arbitrary $\lambda \in \Lambda_{R}^{d}$ and define $\left(\varphi_{s, t}\right)$ by (4.7) and $\left(f_{t}\right)$ by $f_{t}:=g_{t} \circ h_{t}^{-1}$. Then $\left(\varphi_{s, t}\right)$ and $\left(f_{t}\right)$ satisfy conditions (A.1) and (A.2). Moreover, by [17, Lemma 3.2], $\left(f_{t}\right)$ is a Loewner chain of order $d$ associated with the evolution family $\left(\varphi_{s, t}\right)$. This proves that (c) implies (a). 
It remains to show that (b) implies (c). In view of Lemma 4.7 it suffices to verify that to each $t \geq 0$ one can assign a conformal mapping $F_{t}$ of $\mathbb{D}$ onto $\Omega_{t}$ in such a way that $F_{t}^{-1} \circ F_{0} \in \mathcal{C}$ for any $t \geq 0$. According to (A.2) for each $t \geq 0$ there exists $\theta \geq 0$ such that $f_{\theta}(\mathbb{D})=\Omega_{t}$. Note that $f_{0}(\mathbb{D})=\Omega_{0}$ because there can be no two different minimal elements in a linearly ordered set. Hence we can assume that if $t=0$, then $\theta$ also vanishes.

Now take $F_{t}:=f_{\theta}$. Then $F_{t}^{-1} \circ F_{0}=\varphi_{0, \theta} \in \mathcal{C}$ by (A.1). This completes the proof of Statement 1.

4.2.3. Proof of Statement 2 of Theorem 4.8. This statement basically follows from Lemma 4.11. Suppose (e) is true, i.e., there exists $\lambda_{0} \in \Lambda_{R}^{d}$ such that (B) holds for $\lambda:=\lambda_{0}$ with some evolution family $\left(\varphi_{s, t}^{0}\right)$ and some associated Loewner chain $\left(f_{t}^{0}\right)$.

Let us prove that $(\mathrm{d})$ holds. Define a family $\left(\psi_{s, t}\right) \subset \mathrm{Hol}(\mathbb{D}, \mathbb{D})$ by the following formula

$$
\psi_{s, t}:=\left(h_{t}^{0}\right)^{-1} \circ \varphi_{s, t}^{0} \circ h_{s}^{0}, \quad t \geq s \geq 0, \quad h_{t}^{0}(z):=\tau \frac{z-\tau a_{0}(t)}{\tau-a_{0}(t) z}, a_{0}(t):=\frac{e^{\lambda_{0}(t)}-1}{e^{\lambda_{0}(t)}+1}
$$

where $h_{t}^{0}$ coincides with $h_{t}$ given in (4.7) when $\lambda=\lambda_{0}$. We apply Lemma 4.11 for $\lambda:=\lambda_{0}$ to conclude that the family $\left(\psi_{s, t}\right)$ is an evolution family, satisfying condition (ii) in this lemma.

Take now any arbitrary $\lambda \in \Lambda_{R}^{d}$ and use Lemma 4.11 again with this $\lambda$ to construct an evolution family $\left(\varphi_{s, t}\right)$ satisfying condition (i) in this lemma, which implies condition (B.1). Define $\left(f_{t}\right)$ by $f_{t}:=f_{t}^{0} \circ h_{t}^{0} \circ h_{t}^{-1}, t \geq 0$. Note that $f_{t}(\mathbb{D})=f_{t}^{0}(\mathbb{D})$ for all $t \geq 0$. It follows that condition (B.2) is also satisfied. To complete the proof it remains to make sure that $f_{t} \circ \varphi_{s, t}=f_{s}$ for all $s \geq 0$ and $t \geq s$ and apply [17, Lemma 3.2] to conclude that $\left(f_{t}\right)$ is a Loewner chain of order $d$ associated with the evolution family $\left(\varphi_{s, t}\right)$.

\section{L-ADMissible families And Goryainov-Ba Evolution FAMiLiES}

5.1. Definitions and results. Originally the chordal variant of Loewner Theory was developed for the class $\mathcal{H}$ of univalent self-mappings $G$ of the upper half-plane $\mathbb{H}:=\{z$ : $\operatorname{Im} z>0\}$ satisfying the condition

$$
\lim _{\substack{z \rightarrow \infty, z \in \mathbb{H}}}(G(z)-z)=0 .
$$

The parametric representation was given for a dense subclass of $\mathcal{H}$, all elements of which satisfy quite strong regularity condition at the point of infinity, namely $\mathbb{H} \backslash G(\mathbb{H})$ is a bounded set, so that $G$ has meromorphic continuation to a neighbourhood of $\infty$ with real coefficients in its Laurent expansion. The role of Loewner equation (1.1) in this case is played by the so-called chordal Loewner equation

$$
\frac{d w}{d t}=\frac{1}{\lambda(t)-w}, \quad t \geq 0 ;\left.w\right|_{t=0}=z, z \in \mathbb{H},
$$

where $\lambda:[0,+\infty) \rightarrow \mathbb{R}$ is a (piece-wise) continuous driving term. 
Here we cite some papers devoted to this topic without attempts to give the complete bibliography [41, 42, 30, 5, 48]. Mainly the theory was developed as a tool for obtaining functional estimates in the class $\mathcal{H}$.

Much later, the chordal Loewner evolution attracted interest of the wide mathematical society due to the famous paper by Schramm [44], where he introduced and studied stochastic version of Loewner evolution (SLE), based on the simplest variant of radial and chordal Loewner equations. This development led to deep results in mathematical theory of 2D lattice models playing important role in Statistical Physics see, e.g., [31 35].

Here we consider a more general and, in some sense, more natural approach than the one developed in [4, 6, 26], see also [7]. Very close results were independently obtained later by Bauer [8], motivated by a problem connected to the Cauchy transform of probability measures.

For any function $G \in \mathcal{H}$ such that $\mathbb{H} \backslash G(\mathbb{H})$ is bounded, we have

$$
G(z)=z-c / z+\gamma(z), \quad c \geq 0
$$

where $\gamma(z)=o(1 / z)$, i.e.,

$$
\lim _{\substack{z \rightarrow \infty \\ z \in \mathbb{H}}} z \gamma(z)=0
$$

The idea in [4, 6, 26] was to interpret expansion (5.2) in angular sense, i.e., to replace (5.3) with the weaker condition

$$
\angle \lim _{z \rightarrow \infty} z \gamma(z)=0
$$

This leads to the class of functions $\mathfrak{P}_{0}$ characterized in the following way.

Definition 5.1 ([8]). By $\mathfrak{P}_{0}$ we denote the class of all univalent function $G: \mathbb{H} \rightarrow \mathbb{H}$ satisfying the following condition: there exists $C>0$ such that $|G(z)-z| \leq C / \operatorname{Im} z$ for all $z \in \mathbb{H}$. Let us denote by $\ell(G)$ the minimal value of $C$ for which the above estimate holds.

Remark 5.2. Basic facts about the class $\mathfrak{P}_{0}$ can be found in [4, 26, 8]. In particular, the class $\mathfrak{P}_{0}$ is a semigroup with respect to the operation of composition and the functional $\ell$ turns out to be additive, i.e., $\ell\left(G_{1} \circ G_{2}\right)=\ell\left(G_{1}\right)+\ell\left(G_{2}\right), G_{j} \in \mathfrak{P}_{0}, j=1,2$. Moreover, for any $G \in \mathfrak{P}_{0}$ and for any $\varepsilon>0$, we have

$$
\ell(G)=\lim _{\substack{z \rightarrow \infty \\ \operatorname{Im}(z)>\varepsilon}} z(z-G(z))
$$

Another useful fact is that the class $\mathfrak{P}_{0}$ coincides with the class of all univalent functions $F$ in $\mathrm{Hol}(\mathbb{H}, \mathbb{C})$ that admit the following integral representation:

$$
F(z)=z+\int_{\mathbb{R}} \frac{d \mu(x)}{x-z}
$$

where $\mu$ is a finite positive Borel measure on $\mathbb{R}$. 
In [4, 6] and later in [26] there was given a generalization of the original chordal Loewner equation (5.1) for evolution families in the class $\mathfrak{P}_{0}$, with the functional $\ell$ playing the role of the governing parameter.

The fact that the class $\mathfrak{P}_{0}$ can be characterized by expansion (5.2) with the residual term $\gamma$ subject to condition (5.4), follows directly from [4, Proposition 7 and Remark 2].

Now we can switch to the unit disk $\mathbb{D}$ as a reference domain by means of the Cayley map $H$, see formula (3.1). The class $\mathcal{P}_{0}:=\left\{\varphi=H^{-1} \circ G \circ H: G \in \mathfrak{P}_{0}\right\}$ can be also defined as the subclass of $\mathcal{P}$ that consists of all functions $\varphi$ having finite angular derivatives at the point $\tau=1$ up to the third order with $\varphi^{\prime \prime}(1)=0$. In other words, $\varphi \in \mathcal{P}$ belongs to $\mathcal{P}_{0}$ if and only if

$$
\varphi(z)=1+(z-1)-\frac{c(z-1)^{3}}{4}+\gamma(z)
$$

for some $c \in \mathbb{C}$ and $\gamma \in \operatorname{Hol}(\mathbb{D}, \mathbb{C})$ such that

$$
\angle \lim _{z \rightarrow 1} \frac{\gamma(z)}{(z-1)^{3}}=0 .
$$

Suppressing the language, we define $\ell: \mathcal{P}_{0} \rightarrow[0,+\infty)$ by $\ell(\varphi):=\ell\left(H \circ \varphi \circ H^{-1}\right)$. In view of Remark 5.2, it is easy to check by elementary computations that the coefficient $c$ in expansion (5.6) equals $\ell(\varphi)$. It is interesting to mention that the class $\mathcal{P}_{0}$ admits a rigidity property, the so-called Burns-Krantz theorem, see [12], stating that if $\varphi \in \mathcal{P}_{0}$ and $\ell(\varphi)=0$, then $\varphi=\mathrm{id}_{\mathbb{D}}$.

Now we introduce the notion of Goryainov-Ba evolution families, which coincides essentially (but not literally) with those studied by Goryainov, Ba and Bauer.

Definition 5.3. A Goryainov-Ba evolution family is an evolution family $\left(\varphi_{s, t}\right)$ contained in $\mathcal{P}_{0}$ and such that the function $t \mapsto \ell\left(\varphi_{0, t}\right)$ is locally absolutely continuous on $[0,+\infty)$.

Under conditions EF1 and EF2 from Definition 1.1 of evolution families, local absolute continuity of $t \mapsto \ell\left(\varphi_{0, t}\right)$ implies condition EF3. This is the content of the following simple proposition.

Proposition 5.4. Suppose that a family $\left(\varphi_{s, t}\right), s \geq 0, t \geq s$, is contained in $\mathcal{P}_{0}$ and satisfies conditions EF1 and EF2 in Definition 1.1. Let $v(t):=\ell\left(\varphi_{0, t}\right), t \geq 0$. If $v \in A C^{d}([0,+\infty),[0,+\infty))$, then $\left(\varphi_{s, t}\right)$ is a Goryainov-Ba evolution family of order $d$.

Proof. We have only to prove that $\left(\varphi_{s, t}\right)$ satisfies condition EF3, what will be done in the framework of the upper half-plane.

Let us consider the functions

$$
\Phi_{s, t}:=H \circ \varphi_{s, t} \circ H^{-1} .
$$

Note that $\Phi_{s, t} \in \mathfrak{P}_{0}$. It follows that whenever $0 \leq s \leq u \leq t$, we have

$$
\left|\Phi_{s, t}(z)-\Phi_{s, u}(z)\right|=\left|\Phi_{u, t}(\zeta)-\zeta\right| \leq \frac{v(t)-v(u)}{\operatorname{lm} \zeta} \leq \frac{v(t)-v(u)}{\operatorname{Im} z},
$$


where $\zeta:=\Phi_{s, u}(z)$.

Therefore condition EF3 holds with $k_{z, T}:=\left.v^{\prime}\right|_{[0, T]} / \operatorname{Im} z$. This completes the proof.

Our main result in this section is a complete characterization of the geometry of expanding systems of domains which can be obtained as the set of image domains of some Loewner chain associated with a Goryainov-Ba evolution family.

For such a characterization, we introduce the following subclass of the class $\mathcal{C}$ (see Definition 4.4):

$\tilde{\mathcal{C}}:=\left\{\varphi \in \mathcal{C}: \varphi^{(j)}(1) \neq \infty\right.$ exists in angular sense for $j=1,2,3$,

$$
\text { and } \left.\operatorname{Re} \varphi^{\prime \prime}(1)=\varphi^{\prime}(1)\left(\varphi^{\prime}(1)-1\right) / 2\right\} \text {. }
$$

Note that the condition $\operatorname{Re} \varphi^{\prime \prime}(1)=\varphi^{\prime}(1)\left(\varphi^{\prime}(1)-1\right) / 2$ in the above definition is to ensure that the coefficient $b$ in the expansion

$$
\left(H \circ \varphi \circ H^{-1}\right)(z)=a z+b+\frac{c}{z}+\gamma(z), \quad z \in \mathbb{H}, \angle \lim _{z \rightarrow \infty} z \gamma(z)=0,
$$

is a real number for any $\varphi \in \tilde{\mathcal{C}}$.

Theorem 5.5. Let $\left(\Omega_{t}\right)$ be an L-admissible family. Then the following two statements are equivalent:

(i) there exists a Goryainov-Ba evolution family $\left(\varphi_{s, t}\right)$ and a Loewner chain $\left(f_{t}\right)$ associated with $\left(\varphi_{s, t}\right)$ such that

$$
\left\{f_{t}(\mathbb{D}): t \geq 0\right\}=\left\{\Omega_{t}: t \geq 0\right\} ;
$$

(ii) there exist a family $\left(F_{t}\right)$ of univalent functions in the unit disk $\mathbb{D}$ such that

$$
F_{t}(\mathbb{D})=\Omega_{t} \text { and } F_{t}^{-1} \circ F_{0} \in \tilde{\mathcal{C}} \quad \text { for all } t \geq 0 .
$$

Remark 5.6. The evolution family $\left(\varphi_{s, t}\right)$ and the Loewner chain $\left(f_{t}\right)$ we construct in the proof of the "(ii) $\Rightarrow(i)$ "-part of the above theorem, are of order $d=\infty$.

5.2. The class $\mathfrak{P}_{0}$. In order to prove Theorem 5.5 we need to establish some new results about $\mathfrak{P}_{0}$. As usual, for any $b>0$, we write $\mathbb{H}_{b}:=\{w \in \mathbb{H}: \operatorname{Im} w>b\}$.

Lemma 5.7. For each function $F \in \mathfrak{P}_{0}$ there exists $b>0$ such that $\mathbb{H}_{b} \subset F(\mathbb{H})$.

Proof. We will prove a little bit more, namely that $\mathbb{H}_{b} \subset F\left(\mathbb{H}_{1}\right)$. Suppose on the contrary that $\mathbb{H}_{b} \backslash F\left(\mathbb{H}_{1}\right) \neq \emptyset$ for any $b>0$. According to Remark 3.3 ,

$$
\mathbb{H}_{b} \cap F\left(\mathbb{H}_{1}\right) \supset \mathbb{H}_{b} \cap F\left(\mathbb{H}_{b}\right)=F\left(\mathbb{H}_{b}\right) \neq \emptyset
$$

for any $b \geq 1$. It follows that

$$
\mathbb{H}_{b} \cap \partial F\left(\mathbb{H}_{1}\right) \neq \emptyset, \quad \text { for all } b \geq 1 .
$$


Using [13, Theorem 3.1] it is easy to see that $\partial F\left(\mathbb{H}_{1}\right)=\overline{F(\mathbb{L})}$, where $\mathbb{L}:=\{x+i: x \in \mathbb{R}\}$. Consequently, (5.8) implies that $\mathbb{H}_{b} \cap F(\mathbb{L}) \neq \emptyset$ for each $b \geq 1$. It follows that $\operatorname{Im} F$ is not bounded on $\mathbb{L}$.

At the same time, $|F(z)-z| \leq 1 / \operatorname{Im} z=1$ for all $z \in \mathbb{L}$ because $F \in \mathfrak{P}_{0}$. This contradiction proves the lemma.

By [26, Lemma 1], a function $F \in \mathfrak{P}_{0}$ if and only if the following two conditions are satisfied:

$$
\begin{array}{r}
F(i y)-i y \rightarrow 0 \quad \text { as } y \rightarrow+\infty, y>0, \\
\sup _{y>0} y(\operatorname{Im} F(i y)-y)<+\infty .
\end{array}
$$

Using this fact, we easily deduce the following

Lemma 5.8. Let $F: \mathbb{H} \rightarrow \mathbb{H}$ be a univalent holomorphic function. Then the following statements are equivalent:

(i) $F \in \mathfrak{P}_{0}$;

(ii) for each $c \geq 0$ the function $z \mapsto F(z+i c)-i c$ belongs to the class $\mathfrak{P}_{0}$;

(iii) there exists $c \geq 0$ such that the function $z \mapsto F(z+i c)-i c$ belongs to the class $\mathfrak{P}_{0}$.

Proposition 5.9. Let $\varphi_{j} \in \mathcal{P}, j=1,2$,3. If $\varphi_{3}=\varphi_{2} \circ \varphi_{1}$ and $\varphi_{3} \in \mathcal{P}_{0}$, then there exists $h \in \operatorname{Möb}(\mathbb{D}) \cap \mathcal{P}$ such that $h \circ \varphi_{1}$ and $\varphi_{2} \circ h^{-1}$ belong to $\mathcal{P}_{0}$.

Proof. To make the proof easier we reformulate the statement of the proposition in the framework of the upper half-plane. So let $\Phi_{3} \in \mathfrak{P}_{0}, \Phi_{1}, \Phi_{2} \in \mathfrak{P}$ and $\Phi_{3}=\Phi_{2} \circ \Phi_{1}$. We have to prove that there exists a constant $A \in \mathbb{R}$ such that the functions $z \mapsto \Phi_{1}(z)+A$ and $z \mapsto \Phi_{2}(z-A)$ belong $\mathfrak{P}_{0}$.

Since $\Phi_{j} \in \mathcal{P}$, we can write

$$
\Phi_{j}(z)=z+\tilde{\Phi}_{j}(z), \quad j=1,2,3,
$$

where $\tilde{\Phi}_{j}: \mathbb{H} \rightarrow \overline{\mathbb{H}}$ are holomorphic functions satisfying

$$
\angle \lim _{z \rightarrow \infty} \frac{\tilde{\Phi}_{j}(z)}{z}=0 .
$$

This means that the functions $\tilde{\Phi}_{j}$ has vanishing angular derivative at $\infty$. Using Lemma 3.1 one can easily conclude that the same holds for the function $\Psi_{2}:=\tilde{\Phi}_{2} \circ \Phi_{1}$. Therefore, with the notation $\Psi_{3}:=\tilde{\Phi}_{3}, \Psi_{1}:=\tilde{\Phi}_{1}$, we have:

$$
\angle \lim _{z \rightarrow \infty} \frac{\Psi_{j}(z)}{z}=0, \quad j=1,2,3 .
$$

The relation $\Phi_{3}=\Phi_{2} \circ \Phi_{1}$ can be rewritten now in a very simple form

$$
\Psi_{3}=\Psi_{2}+\Psi_{1}
$$


It is known (see, e.g., [2, Vol.2, p. 7-9] or [19, p.18-20]) that every holomorphic function $\Psi: \mathbb{H} \rightarrow \overline{\mathbb{H}}$ can be represented in the following way:

$$
\Phi(z)=\beta z+\alpha+\int_{\mathbb{R}}\left(\frac{1}{x-z}-\frac{x}{1+x^{2}}\right) d \mu(x),
$$

where $\beta \geq 0, \alpha \in \mathbb{R}$, and $\mu$ is a positive Borel measure on $\mathbb{R}$ such that

$$
\int_{\mathbb{R}} d \mu(x) /\left(1+x^{2}\right)<+\infty
$$

All the parameters in representation (5.13) $, \alpha, \beta$, and $\mu$ are defined by the function $\Phi$ in a unique way.

Denote by $V(z, x)$ the integrand in (5.13). Obviously, $V(z, x) / z \rightarrow 0$ as $z \rightarrow \infty$ pointwise w.r.t. $x \in \mathbb{R}$. Moreover, it is easy to check that

$$
|V(z, x) / z| \leq \frac{1}{1+x^{2}}\left[\frac{1}{|z| \operatorname{Im} z}+\frac{\operatorname{Re} z}{\operatorname{Im} z}+1\right]
$$

for all $x \in \mathbb{R}$ and $z \in \mathbb{H}$. Therefore, according to the Lebesgue dominated convergence theorem, $(1 / z) \int_{\mathbb{R}} V(z, x) d \mu(x) \rightarrow 0$ as $z$ tends to $\infty$ within any Stolz angle

$$
\Delta_{\varepsilon}:=\{z: \operatorname{lm} z>\varepsilon|z|\}, \quad \varepsilon>0 .
$$

Hence we conclude that the parameter $\beta$ in (5.13) equals the angular derivative of $\Phi$ at $\infty$.

Now let us write representation (5.13) for the functions $\Psi_{j}$. According to (5.11), it will have the following form:

$$
\Psi_{j}(z)=\alpha_{j}+\int_{\mathbb{R}}\left(\frac{1}{x-z}-\frac{x}{1+x^{2}}\right) d \mu_{j}(x) .
$$

On the other hand, $\Phi_{3} \in \mathfrak{P}_{0}$. Consequently, by Remark 5.2,

$$
\Psi_{3}(z)=\int_{\mathbb{R}} \frac{d \mu_{0}(x)}{x-z}
$$

for some finite measure Borel measure on $\mathbb{R}$. It follows that

$$
\mu_{3}=\mu_{0}, \quad \alpha_{3}=\int_{\mathbb{R}} \frac{x}{1+x^{2}} d \mu_{0}(x) .
$$

Furthermore, according to the uniqueness of $\alpha_{j}$ and $\mu_{j}$ in representation (5.14), equality (5.12) implies that

$$
\mu_{3}=\mu_{2}+\mu_{1}, \quad \alpha_{3}=\alpha_{2}+\alpha_{1} .
$$

In particular, the measures $\mu_{1}$ and $\mu_{2}$ are finite. Combining (5.15) with (5.16) we conclude that

$$
A:=\alpha_{2}-\int_{\mathbb{R}} \frac{x}{1+x^{2}} d \mu_{2}(x)=-\alpha_{1}+\int_{\mathbb{R}} \frac{x}{1+x^{2}} d \mu_{1}(x) .
$$

Now the fact that $\Phi_{1}+A \in \mathfrak{P}_{0}$ follows from (5.14) and Remark 5.2. 
Let $z \in \mathbb{H}$. Denote $w:=\Phi_{1}(z)+A$. Then $\Phi_{2}(w-A)-w=\Phi_{3}(z)-z-\left(\Phi_{1}(z)+A-z\right)$. It follows that

$$
\left|\Phi_{2}(w)-w\right| \leq \frac{C}{\operatorname{Im} z} \leq \frac{C}{\operatorname{Im} w}
$$

for some $C>0$, which does not depend on $z$. Since $z \in \mathbb{H}$ is arbitrary, inequality (5.17) holds for all $w$ such that $w-A \in \Phi_{1}(\mathbb{H})$. Since $\Phi_{1} \in \mathfrak{P}_{0}$, by Lemma 5.7 there exists $b>0$ such that $\mathbb{H}_{b} \subset \Phi_{1}(\mathbb{H})$. Hence (5.17) holds for all $w \in \mathbb{H}_{b}$, which can be reformulated in the following way: inequality (5.17) holds for all $w \in \mathbb{H}$ and $\Phi_{2}$ replaced by the function $\Phi_{2, b}(w):=\Phi_{2}(w+i b)-i b$. Hence $\Phi_{2, b} \in \mathfrak{P}_{0}$. But by Lemma 5.8 the latter statement implies that the function $\Phi_{2}$ also belongs to the class $\mathfrak{P}_{0}$. The proof is now finished.

Corollary 5.10. Suppose that $\varphi_{j}, j=1,2,3$, are univalent self-mappings of $\mathbb{D}$, and $\varphi_{3}=\varphi_{2} \circ \varphi_{1}$. If any two of these three functions belong to $\mathcal{P}_{0}$, then so does the third one.

Proof. If $\varphi_{1}, \varphi_{2} \in \mathcal{P}_{0}$, then $\varphi_{3}$ also belongs to $\mathcal{P}_{0}$ because of the semigroup property of the class $\mathfrak{P}_{0}$, see Remark 5.2 ,

Suppose now that $\varphi_{3} \in \mathcal{P}_{0}$ and one of the functions $\varphi_{1}, \varphi_{2}$ belongs to $\mathcal{P}_{0}$. Then by Lemma 3.4, we have $\varphi_{j} \in \mathcal{P}$ for $j=1,2,3$. Now one can apply Proposition 5.9 to deduce that $\varphi_{j} \in \mathcal{P}_{0}$ for $j=1,2$, which finishes the proof.

Another tool in the proof of Theorem 5.5 is [8, Theorem 4.2], which, in particular, contains the following

Proposition 5.11. Let $\left(G_{n}\right)$ be a sequence from $\mathfrak{P}_{0}$ such that for any $n, m \in \mathbb{N}$ either $G_{n}(\mathbb{H}) \subset G_{m}(\mathbb{H})$, or $G_{n}(\mathbb{H}) \supset G_{m}(\mathbb{H})$. If $\ell\left(G_{n}\right)$ has a finite limit as $n \rightarrow+\infty$, then $G_{n}$ converges to a function $G \in \mathfrak{P}_{0}$ uniformly on $\mathbb{H}_{\varepsilon}:=\{z: \operatorname{Im} z>\varepsilon\}$ for each $\varepsilon>0$, with

$$
\begin{gathered}
\ell(G)=\lim _{n \rightarrow+\infty} \ell\left(G_{n}\right), \\
G(\mathbb{H})=\bigcup_{\varepsilon>0} \bigcup_{k \in \mathbb{N}} \operatorname{int}\left(\bigcap_{n>k} G_{n}\left(\mathbb{H}_{\varepsilon}\right)\right) .
\end{gathered}
$$

Remark 5.12. If $F, G \in \mathfrak{P}_{0}$ and $F(\mathbb{H}) \subset G(\mathbb{D})$, then $F=G \circ W$, where $W=G^{-1} \circ F$ is a conformal mapping of $\mathbb{H}$ into itself. It is actually known (see, e.g., [8, Lemma 4.1]) that $W \in \mathfrak{P}_{0}$. Therefore, according to Remark 3.3, $W\left(\mathbb{H}_{\varepsilon}\right) \subset \mathbb{H}_{\varepsilon}$ for any $\varepsilon>0$. As a consequence, the inclusion $F(\mathbb{H}) \subset G(\mathbb{H})$, where $F, G \in \mathfrak{P}_{0}$, implies that $F\left(\mathbb{H}_{\varepsilon}\right) \subset G\left(\mathbb{H}_{\varepsilon}\right)$, for any $\varepsilon>0$. It also follows that for $F, G \in \mathfrak{P}_{0}$ the equality $F(\mathbb{H})=G(\mathbb{H})$ occurs only if $F=G$.

5.3. Proof of Theorem 5.5. First of all let us note that the implication $(i) \Rightarrow(i i)$ is almost trivial. Indeed, assume (i) holds and find for each $t \geq 0$ a number $\theta=\theta(t) \geq 0$ such that $f_{\theta(t)}(\mathbb{D})=\Omega_{t}$. We can assume that $\theta(0)=0$. Then functions $F_{t}:=f_{\theta(t)}$ form the family one have to construct in order to prove (ii). 
Let us now assume that (ii) holds. We are going to prove (i). To obtain this implication we switch to the upper half-plane $\mathbb{H}$ as a reference domain. So assume that for each $t \geq 0$ the function $F_{t}$ maps $\mathbb{H}$ conformally onto $\Omega_{t}$ and

$$
\left(F_{t}^{-1} \circ F_{0}\right)(z)=a(t) z+b(t)+\frac{c(t)}{z}+\gamma_{t}(z), \quad \angle \lim _{z \rightarrow \infty} z \gamma_{t}(z)=0,
$$

where $a(t)>0$ and $b(t) \in \mathbb{R}$.

The proof follows the same scheme as for Theorem 4.10. First of all we take Möbius transformations $L_{t}(z):=a(t) z+b(t)$ and define $H_{t}:=F_{t} \circ L_{t}$. Denote $\Phi_{s, t}:=H_{t}^{-1} \circ H_{s}$. We claim that

$$
\Phi_{s, t} \in \mathfrak{P}_{0}, \quad \text { for all } s \geq 0, t \geq s .
$$

Indeed, for $s=0$ the above assertion follows from (5.20) and the fact that $a(0)=1$, $b(0)=0$. By the same reason $\Phi_{0, s} \in \mathfrak{P}_{0}$. Now (5.21) follows from the equality $\Phi_{s, t} \circ \Phi_{0, s}=$ $\Phi_{0, t}$ and Corollary 5.10.

Let us denote $x(t):=\ell\left(\Phi_{0, t}\right), t \geq 0$. Since the functional $\ell$ is additive,

$$
x(t)=x(s)+\ell\left(\Phi_{s, t}\right) \quad \text { for all } s \geq 0 \text { and } t \geq s .
$$

By [8, Lemma 4.1], it follows that $t \mapsto x(t)$ is non-decreasing and

$$
x(t)=x(s) \Longleftrightarrow \Phi_{s, t}=\mathrm{id}_{\mathbb{H}} \text {. }
$$

Fixing the parameter $t$ and regarding the parameter $s$ in equality (5.22) as a variable, one can use Proposition 5.11 along with Remarks 1.7 and 5.12 to see that the function $[0,+\infty) \ni t \mapsto x(t) \in[0,+\infty)$ is continuous.

Let us now set $h_{t}:=H_{t} \circ H, \phi_{s, t}:=H^{-1} \circ \Phi_{s, t} \circ H, t \geq 0, s \in[0, t]$, where $H$ stands for the Cayley map (3.1) sending of $\mathbb{D}$ onto $\mathbb{H}$ with $H(0)=i$ and $H(1)=\infty$. The rest of the proof is almost the same as Steps 3 and 4 in the proof of Theorem 4.10, except that $x(t)$ can be now unbounded and instead of Proposition 4.9 we apply Proposition 5.4. By this reason, we omit the details.

\section{SufFICIENT CONDITIONS FOR CHORDAL ADMISSIBILITY}

The condition of conformal embedding at a prime end, contained in the definition of chordally admissible families of simply connected domains, involves in an essential way information on boundary behaviour of conformal mappings related to these domains. This might make direct application of Theorem 4.8 in many cases quite difficult. That is why it is interesting to establish some sufficient conditions for chordal admissibility of purely geometric nature. An example of such a sufficient condition is the following theorem.

A closed Jordan curve $C \subset \mathbb{C}$ in the complex plane $\mathbb{C}$ is said to be Dini-smooth if there exists a bijective mapping $h$ of $\mathbb{T}:=\partial \mathbb{D}$ onto $C$ such that the function $\mathbb{R} \ni \theta \mapsto h_{1}(\theta):=$ $h\left(e^{i \theta}\right) \in C$ has non-vanishing Dini-continuous derivative. A closed Jordan curve $C$ in the Riemann sphere $\overline{\mathbb{C}}$ is called Dini-smooth if for some (or equivalently for all) Möbius transformation $T$ sending $C$ into $\mathbb{C}$, the image $T(C)$ of $C$ is a Dini-smooth curve. 
Theorem 6.1. Let $\left(\Omega_{t}\right)$ be an L-admissible family and $p \in \partial_{\infty} \Omega_{0}$. Suppose that the following conditions hold:

(i) there exists a Dini-smooth closed Jordan curve $C$ in the Riemann sphere such that $p \in C$ and one of the two connected components of $\overline{\mathbb{C}} \backslash C$ is contained in $\Omega_{0}$.

(ii) for each $t>0$ there exists a Dini-smooth closed Jordan curve $C_{t}$ in the Riemann sphere such that $p \in C_{t}$ and $\Omega_{t} \cap C_{t}=\emptyset$.

Then the family $\left(\Omega_{t}\right)$ is chordally admissible.

Proof. Denote by $D$ the connected component of $\overline{\mathbb{C}} \backslash C$ that is contained in $\Omega_{0}$. Since $D$ is a Jordan domain, there exists a conformal mapping $f_{1}$ of $\mathbb{D}$ onto $D$ continuously extandable to $\partial \mathbb{D}$ with $f_{1}(1)=p$. Let us denote by $\Gamma$ the curve $[0,1) \ni x \mapsto f_{1}(x) \in D \subset \Omega_{0}$. Obviously, we have $\Gamma(x) \rightarrow p$ as $x \rightarrow 1-$.

We note that since $p \in \partial_{\infty} \Omega_{0}$, condition $(i i)$ in the theorem implies that $p \in \partial_{\infty} \Omega_{t}$ for all $t \geq 0$.

The remaining part of the proof will be divided into three steps.

Step 1. For each $t \geq 0$ there exists a conformal mapping $F_{t}$ of $\mathbb{D}$ onto $\Omega_{t}$ such that the following two angular limits exist in the Riemann sphere:

$$
\begin{aligned}
& \angle \lim _{z \rightarrow 1} F_{t}(z)=p, \\
& \angle \lim _{z \rightarrow 1} \Phi_{t}(z)=1, \quad \text { where } \Phi_{t}:=F_{t}^{-1} \circ F_{0} .
\end{aligned}
$$

Proof of Step 1: Fix $t \geq 0$ and let $G_{t}$ be any conformal mapping of $\mathbb{D}$ onto $\Omega_{t}$. Bearing in mind that $p \in \partial_{\infty} \Omega_{t}$ and that $G_{t}$ is univalent, we find in accordance with Theorem E that the curve $\gamma_{t}:[0,1) \rightarrow \mathbb{D} ; x \mapsto G_{t}^{-1}(\Gamma(x))$ lands at some particular point $\xi_{t} \in \mathbb{T}$, i.e., $\gamma_{t}(x) \rightarrow \xi_{t}$ as $x \rightarrow 1-$. Note also that $G_{t}\left(\gamma_{t}(x)\right)=\Gamma(x) \rightarrow p$ as $x \rightarrow 1-$. Therefore, $p$ is an asymptotic value for $G_{t}$ at the point $\xi_{t}$. Since $G_{t}$ is univalent, it is also a normal function (see [39, Lemma 9.3]). Therefore, by Lehto-Virtanen's Theorem (see, e.g., [39, Theorem 9.3]) $G_{t}$ has angular limit at $\xi_{t}$ equal to $p$.

Now, define $F_{t}(z):=G_{t}\left(\xi_{t} z\right), z \in \mathbb{D}$. Statement (6.1) clearly holds. To deal with statement (6.2), we fix $t>0$. Likewise, we denote $\gamma(x):=F_{0}^{-1}(\Gamma(x))$. Appealing again to Theorem E, we conclude that $\gamma$ is a Jordan arc with $\gamma(x) \rightarrow 1$ as $x \rightarrow 1-$. Now, note that $\Phi_{t}(\gamma(x))=\overline{\xi_{t}} \gamma_{t}(x)$. Hence, $\Phi_{t}$ has an asymptotic value at $z=1$, equal to 1 . Once again, by [39, Lemma 9.3] and Lehto-Virtanen's Theorem, we conclude that the angular limit of $\Phi_{t}$ at the point $z=1$ equals 1 .

Step 2. For each $t>0$ the function $\Phi_{t}$ has finite angular derivative at the point $z=1$.

Observe that $\Phi_{t}$ is a holomorphic self-map of the unit disk having an (angular) fixed point at $z=1$ by (6.2). According to Remark 3.2 , the following limit exists

$$
\Phi_{t}^{\prime}(1):=\angle \lim _{z \rightarrow 1} \frac{\Phi_{t}(z)-1}{z-1} \in(0,+\infty) \cup\{\infty\} .
$$


Further we proceed by contradiction. So we assume that for some $t>0$ we have $\Phi_{t}^{\prime}(1)=\infty$. Since $\Phi_{t}$ is univalent, one can again take advantage of (6.2) and [39, Theorem 10.5 on p. 305] to conclude that $\angle \lim _{z \rightarrow 1} \Phi_{t}^{\prime}(z)=\infty$.

Since $C_{t} \cap \Omega_{t}=\emptyset$, the domain $\Omega_{t}$ is contained in one of the connected components of $\overline{\mathbb{C}} \backslash C_{t}$, which we denote by $D_{t}$. Applying if necessary the Möbius transformation $T(z):=1 /\left(z-z_{0}\right)$, where $z_{0} \in \mathbb{C}$ is any point in the exterior of $D_{t}$, we can assume that $D_{t} \subset \mathbb{C}$ and is a bounded domain. Then $D$ is also bounded, with $\partial D=C$ being a Jordan curve in the plane. Note that applying $T$ will change $f_{1}, F_{0}$ and $F_{t}$, but not the function $\Phi_{t}$.

Since by condition (i), $C$ is a Dini-smooth Jordan curve, from [40, Theorem 3.5] it follows that there exists finite angular derivative $f_{1}^{\prime}(1) \neq 0$. At the same time,

$$
f_{1}(\mathbb{D})=D \subset \Omega_{0}=F_{0}(\mathbb{D}) .
$$

Recall that $F_{0}(1)=p$ in the angular sense. Applying [40, Theorem 4.14], we conclude that $F_{0}$ has finite angular derivative at $z=1$. Since $F_{0}$ is univalent, we have (see again [39, Theorem 10.5])

$$
F_{0}^{\prime}(1)=\lim _{r \rightarrow 1-} F_{0}^{\prime}(r) \in \mathbb{C} .
$$

Differentiating the identity $F_{t} \circ \Phi_{t}(r)=F_{0}(r), r \in(0,1)$, and passing to limits as $r \rightarrow 1-$, we deduce that $\lim _{r \rightarrow 1-} F_{t}^{\prime}\left(\Phi_{t}(r)\right)=0$. Now, by (6.2) we see that $r \in[0,1) \mapsto \Phi_{t}(r) \in \mathbb{D}$ is a Jordan arc in $\mathbb{D}$ landing at $z=1$. Therefore, 0 is an asymptotic value of $F_{t}^{\prime}$ at the point $z=1$. Since $F_{t}$ is univalent, its derivative $F_{t}^{\prime}$ is a normal function (see, e.g., [39, Lemma 9.3]). Therefore, from Lehto-Virtanen's Theorem (see, e.g., [39, Theorem 9.3]) it follows that $\angle \lim _{z \rightarrow 1} F_{t}^{\prime}(z)=0$.

Now let us consider the domain $D_{t}$. We argue in a similar way as above. Namely, we let $f_{2}$ be any conformal mapping of $\mathbb{D}$ onto $D_{t}$. The $C_{t}=\partial D_{t}$ is a Dini-smooth Jordan curve in $\mathbb{C}$ and $p \in C_{t}$. Therefore, from [40, Theorem 3.5], it follows that $f_{2}\left(\zeta_{2}\right)=p$ for some (in fact the unique one) $\zeta_{2} \in \mathbb{T}$ and that there exists finite angular derivative $f_{2}^{\prime}\left(\zeta_{2}\right) \neq 0$. Recall that $F_{t}(1)=p$ in the angular sense. Therefore, applying [40, Theorem 4.14], we conclude that $\left|f_{2}^{\prime}\left(\zeta_{2}\right)\right|=c\left|F_{t}^{\prime}(1)\right|$ for some $c>0$. However, the left-hand side of this equality is different from 0 while the right-hand side vanishes. This contradiction proves Step 2.

Step 3. The family $\left(\Omega_{t}\right)$ is chordally admissible.

Just apply Lemma 4.7 using previous Step 2.

Now let us formulate an analog of Theorem 6.1 for Goryainov-Ba evolution families. Let us introduce some definitions.

Let $n \in \mathbb{N}$ and $\alpha \in(0,1)$. A closed Jordan curve $C$ in the complex plane $\mathbb{C}$ will be called $C^{n,+0}$-smooth if there is a bijective mapping $h$ of $\mathbb{T}:=\partial \mathbb{D}$ onto $C$ such that the function $\mathbb{R} \ni \theta \mapsto h_{1}(\theta):=h\left(e^{i \theta}\right) \in C$ has derivatives up to the order $n$, the first derivative $h_{1}^{\prime}$ does not vanish, and $h_{1}^{(n)}$ is Hölder continuous with some exponent $\alpha>0$. A closed Jordan 
curve $C$ in the Riemann sphere $\overline{\mathbb{C}}$ is called $C^{n,+0}$-smooth if for some (or equivalently for all) Möbius transformation $T$ sending $C$ into $\mathbb{C}$, the image $T(C)$ of $C$ is a $C^{n,+0}$-smooth curve.

Two $C^{1}$-smooth Jordan curves $C_{1}, C_{2} \in \mathbb{C}$ with a common point $p$ is said to have contact of order $n \in \mathbb{N}$ at the point $p$, if there exist $C^{1}$-smooth parameterizations $w_{1}:[-1,1] \rightarrow \mathbb{C}$, $w_{2} \in[-1,1] \rightarrow \mathbb{C}$ of the curves $C_{1}$ and $C_{2}$, respectively, such that $w_{1}(0)=w_{2}(0)=p$, $w_{1}^{\prime}(0), w_{2}^{\prime}(0) \neq 0$, and $\left|w_{1}(t)-w_{2}(t)\right|=o\left(t^{n}\right)$ as $t \rightarrow 0$. Again using Möbius transformations one can extend this definition to the case of Jordan curves in the Riemann sphere and $p=\infty$.

Theorem 6.2. Let $\left(\Omega_{t}\right)$ be an L-admissible family and $p \in \partial_{\infty} \Omega_{0}$. Suppose that the following conditions hold:

(i) there exists a $C^{3,+0}$-smooth closed Jordan curve $C$ in the Riemann sphere such that $p \in C$ and one of the two connected components of $\overline{\mathbb{C}} \backslash C$ is contained in $\Omega_{0}$.

(ii) for each $t>0$ there exists a $C^{3,+0}$-smooth closed Jordan curve $C_{t}$ in the Riemann sphere such that

(ii.1) $p \in C_{t}$,

(ii.2) the curves $C$ and $C_{t}$ have second order contact at the point $p$, and

(ii.3) $\Omega_{t} \cap C_{t}=\emptyset$.

Then the family $\left(\Omega_{t}\right)$ satisfies condition (ii) in Theorem 5.5 .

For the proof of the above theorem we need the following elementary lemmas.

Lemma 6.3. Let $\varphi: \mathbb{D} \rightarrow \mathbb{D}$ be a holomorphic univalent function having $C^{3}$-smooth injective extension to $\partial \mathbb{D}$ with $\varphi(1)=1$. If the curve $\partial \varphi(\mathbb{D})$ has second order contact with $\partial \mathbb{D}$ at the point $z=1$, then there exists $h \in \operatorname{Möb}(\mathbb{D})$ such that the function $h \circ \varphi$ belongs to the class $\mathcal{P}_{0}$.

Proof. This statement is easier to prove if we pass to the upper half-plane. So we consider the function $\Phi:=H \circ \varphi \circ H^{-1}$, where as earlier $H$ stands for the Cayley map (3.1). Using the facts that $\varphi$ has $C^{3}$-smooth extension to $\partial \mathbb{D}$ and that $\varphi(1)=1$, we can assume that the following expansion takes place:

$$
\Phi(z)=a z+b+\frac{c}{z}+\gamma(z), z \in \mathbb{H} \cup \mathbb{R}, \quad a \neq 0, \lim _{\substack{z \rightarrow \infty, z \in \mathbb{H} \cup \mathbb{R}}} z \gamma(z)=0 .
$$

The fact that $\partial \varphi(\mathbb{D})$ has second order contact with $\partial \mathbb{D}$ at $z=1$ can be reformulated in the following way:

$$
\operatorname{Im} \Phi(t) \rightarrow 0 \quad \text { as } \quad t \rightarrow \infty, t \in \mathbb{R} .
$$

(The proof of this claim is elementary and so we omit it.)

Combining (6.3) with (6.4) and taking into account that $\Phi(\mathbb{H}) \subset \mathbb{H}$, we conclude that $a>0$ and $b \in \mathbb{R}$. Denote $L(z):=(z-b) / a$. It follows that $L \circ \Phi \in \mathfrak{P}_{0}$. Consequently, $h \circ \varphi \in \mathcal{P}_{0}$, where $h:=H^{-1} \circ L \circ H$, which completes the proof. 
Lemma 6.4. Let $D_{1}$ and $D_{2}$ be domains in the Riemann sphere bounded by $C^{3,+0}$-smooth Jordan curves $C_{1}$ and $C_{2}$ respectively. Suppose that $D_{1} \subset D_{2}$ and that the intersection $C_{1} \cap C_{2}$ contains a point $p$ at which the curves $C_{1}$ and $C_{2}$ have contact of second order. If $f_{1}$ maps conformally $\mathbb{D}$ onto $D_{1}$ in such a manner that $f_{1}(1)=p$, then there exists a conformal mapping $f_{2}$ of $\mathbb{D}$ onto $D_{2}$ such that the function $f_{2}^{-1} \circ f_{1}$ belongs to the class $\mathcal{P}_{0}$.

Proof. First of all we note that because of the smoothness of the curves $C_{1}$ and $C_{2}$, any conformal mappings $f_{1}$ and $f_{2}$ of $\mathbb{D}$ onto $D_{1}$ and $D_{2}$ respectively, have $C^{3, \alpha_{\text {-smooth }}}$ extension to the unit circle with some $\alpha>0$, see, e.g., [40, Theorem 3.6]. Assume now, according to the conditions of the lemma, that $f_{1}(1)=p$. We also can choose $f_{2}$ in such a way that $f_{2}(1)=p$. Then the function $\varphi:=f_{2}^{-1} \circ f_{1}$ has $C^{3}$-smooth extension to $\partial \mathbb{D}$ and maps $\mathbb{D}$ onto a subdomain of $\mathbb{D}$ bounded by a curve having second order contact point with $\partial \mathbb{D}$ at $z=1$. Then by Lemma 6.3, $h \circ \varphi \in \mathcal{P}_{0}$ for some $h \in \operatorname{Möb}(\mathbb{D})$. Hence, we may finish the proof by replacing $f_{2}$ with $f_{2} \circ h^{-1}$.

Lemma 6.5. Let $U \subset \mathbb{D}$ be a simply connected domain and $\varphi \in \mathcal{P}$. If $\varphi(\mathbb{D}) \subset U$, then there exists $\varphi_{2} \in \mathcal{P}$ such that $\varphi_{2}(D)=U$.

Proof. Let $f_{2}$ be any conformal mapping of $\mathbb{D}$ onto $U$ and $\Gamma:=\varphi([0,1))$. Then the curve $\Gamma$ lands at the point $z=1$, which is a common boundary point for the domains $\varphi(\mathbb{D})$ and $U$. According to Theorem $\mathrm{E}$ the curve $\Gamma_{2}:=f_{2}^{-1}(\Gamma)$ lands at some particular point $\zeta_{2} \in \partial \mathbb{D}$. Now we can apply [40, Theorem 4.14] for $f_{1}:=\varphi$ to deduce that there exists finite angular derivative $f_{2}^{\prime}\left(\zeta_{2}\right)$ and therefore there exists the angular limit $\angle \lim _{\zeta \rightarrow \zeta_{2}} f_{2}(\zeta)$, which equals 1 because by just mentioned Theorem E the image of $\Gamma^{\prime}:=f_{2}([0,1))$ can not land on $\partial f_{2}(\mathbb{D})$ at a point different from the landing point of $\Gamma=f_{2}\left(\Gamma_{2}\right)$.

Let us consider the function $g(z):=f_{2}\left(z / \zeta_{2}\right)$. There exist $g(1)=1$ and $g^{\prime}(1) \neq \infty$ in angular sense. Moreover, according to Remark [3.2, $g^{\prime}(1)>0$. To complete the proof it suffices now to set $\varphi_{2}:=g \circ \ell_{x}$, where

$$
\ell_{x}(z):=\frac{z-x}{1-x z}, \quad x:=\frac{1-g^{\prime}(1)}{1+g^{\prime}(1)}
$$

Proof of Theorem [6.2. Denote by $D$ the connected component of $\overline{\mathbb{C}} \backslash C$ that is contained in $\Omega_{0}$. Since $D$ is a Jordan domain, there exists a conformal mapping $F$ of $\mathbb{D}$ onto $D$ continuously extendable to $\partial \mathbb{D}$ with $F(1)=p$.

Fix any $t>0$. Since $C_{t}$ does not intersect $\Omega_{t}$, one of the connected components of $\overline{\mathbb{C}} \backslash C_{t}$ contains $\Omega_{t}$. Denote this connected component by $D_{t}$ and apply Lemma 6.4 with $F$, $D$, and $D_{t}$ substituted for $f_{1}, D_{1}$, and $D_{2}$, respectively. By this lemma there exists a conformal mapping $f_{2}$ of $\mathbb{D}$ onto $D_{t}$ such that $\varphi:=f_{2}^{-1} \circ F$ belongs to $\mathcal{P}_{0}$. By construction $D \subset \Omega_{0} \subset \Omega_{t} \subset f_{2}(\mathbb{D})$. Let $U:=f_{2}^{-1}\left(\Omega_{t}\right)$ and $U_{0}:=f_{2}^{-1}\left(\Omega_{0}\right)$. Note that $\varphi(\mathbb{D}) \subset U_{0} \subset U$. Then by Lemma 6.5 there exist $\varphi_{2}, \varphi_{2}^{0} \in \mathcal{P}$ mapping $\mathbb{D}$ conformally onto $U$ and $U_{0}$, respectively. Now using Lemma 3.4 we conclude that $\varphi=\varphi_{2}^{0} \circ \varphi_{1}^{0}$ and $\varphi_{2}^{0}=\varphi_{2} \circ \varphi_{1}$ for 
some functions $\varphi_{1}, \varphi_{1}^{0} \in \mathcal{P}$. By Proposition 5.9 with $\varphi, \varphi_{2}^{0}$, and $\varphi_{1}^{0}$ substituted for $\varphi_{3}$, $\varphi_{2}$, and $\varphi_{1}$, respectively, there exists $h_{0} \in \operatorname{Möb}(\mathbb{D}) \cap \mathcal{P}$ such that the functions $h_{0} \circ \varphi_{1}^{0}$ and $\varphi_{2}^{0} \circ h_{0}^{-1}$ belong to the class $\mathcal{P}_{0}$. Finally, we apply Proposition 5.9 with $\varphi_{2}^{0} \circ h_{0}^{-1}, \varphi_{2}$, and $\varphi_{1} \circ h_{0}^{-1}$ substituted for $\varphi_{3}, \varphi_{2}$, and $\varphi_{1}$, respectively, to conclude that there exists $h \in \operatorname{Möb}(\mathbb{D}) \cap \mathcal{P}$ such that $h \circ \varphi_{1} \circ h_{0}^{-1} \in \mathcal{P}_{0}$.

Define $F_{t}^{0}:=f_{2} \circ \varphi_{2}^{0} \circ h_{0}^{-1}$ and $F_{t}:=f_{2} \circ \varphi_{2} \circ h^{-1}$. These functions are conformal mappings of $\mathbb{D}$ onto domains $\Omega_{0}$ and $\Omega_{t}$, respectively, with

$$
F_{t}^{-1} \circ F_{t}^{0}=h \circ \varphi_{1} \circ h_{0}^{-1} \in \mathcal{P}_{0} .
$$

One more important relation follows from the above construction. Namely, denote $\varphi_{t}:=h_{0} \circ \varphi_{1}^{0}$. Then

$$
F_{t}^{0} \circ \varphi_{t}=F .
$$

We have constructed a family $\left(F_{t}^{0}\right)$ of conformal mappings of $\mathbb{D}$ onto $\Omega_{0}$. Let us prove that $F_{t}^{0}$ does not depend on $t$. To this end take arbitrary $s, t>0$ and write $h_{s, t}:=\left(F_{t}^{0}\right)^{-1} \circ F_{s}^{0}$. This function maps $\mathbb{D}$ conformally onto itself, i.e., $h_{t} \in \operatorname{Möb}(\mathbb{D})$. Now recall that $F$ does not depend on $t$. According to (6.6), it follows that $\varphi_{t}=h_{s, t} \circ \varphi_{s}$. Recall also that $\varphi_{t} \in \mathcal{P}_{0}$. Therefore, by Corollary 5.10 with $\varphi_{t}, h_{s, t}$, and $\varphi_{s}$ substituted for $\varphi_{3}, \varphi_{2}$, and $\varphi_{1}$, respectively, we have $h_{s, t} \in \mathcal{P}_{0}$. Since $\operatorname{Möb}(\mathbb{D}) \cap \mathcal{P}_{0}=\left\{\operatorname{id}_{\mathbb{D}}\right\}$, we conclude that $h_{s, t}=\mathrm{id}_{\mathbb{D}}$ and therefore $F_{s}^{0}=F_{t}^{0}$. Now in view of (6.5) we may finish the proof just by setting $F_{0}:=F_{t}^{0}$.

The cases when Theorems 6.1 and 6.2 can be applied are quite different from the classical setting of slit mappings. The following corollary of Theorem 5.5 is a simple assertion covering, in an obvious way, the case of an L-admissible family obtained by erasing a slit in the complex plane or in the half-plane. More general case of a slit in an arbitrary simply connected domain can be treated by using a conformal mapping onto the half-plane.

In the formulation and the proof we use the theory of primes ends. The main definitions and some basic facts from this theory can be found, e.g., in [13, Chapter 9] or [40, Chapter 2].

Corollary 6.6. Let $\left(\Omega_{t}\right)$ be an L-admissible family and $P_{0}$ any prime end of the domain $\Omega_{0}$. Suppose that $P_{0}$ is degenerate, i.e., the impression of $P_{0}$ consists of one point $p_{0}$, and for each $t \geq 0$ there exists $\varepsilon>0$ such that

$$
\partial \Omega_{t} \cap D\left(p_{0}, \varepsilon\right)=\partial \Omega_{0} \cap D\left(p_{0}, \varepsilon\right),
$$

where $D\left(p_{0}, \varepsilon\right)$ stands for the disk of radius $\varepsilon$ centered at $p_{0}$. Then assertions (i) and (ii) in Theorem 5.5 hold and, in particular, the family $\left(\Omega_{t}\right)$ is chordally admissible.

Proof. Let $F_{0}$ be a conformal mapping of $\mathbb{D}$ onto $\Omega_{0}$. The fact that the impression $I\left(P_{0}\right)$ of the prime end $P_{0}$ consists of only one point $p_{0}$, implies that $F_{0}$ has unrestricted limit 
equal to $p_{0}$ at some point $\zeta_{0} \in \mathbb{T}$ and the curve $F_{0}\left(\zeta_{0}[0,1)\right)$ tends to $P_{0}$. Using rotation of $\mathbb{D}$ we can assume that $\zeta_{0}=1$.

Now let us fix $t>0$ and let $F_{t}$ be a conformal mapping of $\mathbb{D}$ onto $\Omega_{t}$. Another consequence of the degeneracy of $P_{0}$ is that for any $\varepsilon>0$ there exists a null-chain $\left(C_{n}\right)_{n \in \mathbb{N}}$ belonging to the prime end $P_{0}$ such that for any $n \in \mathbb{N}$ the connected component $V_{n}$ of the set $\Omega_{0} \backslash C_{n}$ containing $C_{n+1}$, lies in $D\left(p_{0}, \varepsilon\right)$ along with its boundary. Indeed, take any null-chain $\left(C_{n}\right)_{n=0}^{+\infty}$ belonging to the prime end $P_{0}$. Since by definition of the impression of prime end,

$$
\left\{p_{0}\right\}=I\left(P_{0}\right)=\bigcap_{n \in \mathbb{N}} \overline{V_{n}}
$$

we have $V_{n} \subset D\left(p_{0}, \varepsilon\right)$ for all $n$ sufficiently large. Therefore, dropping a finite number of cross-cuts $C_{n}$ we get the desired null-chain with all $\overline{V_{n}}$ lying in $D\left(p_{0}, \varepsilon\right)$.

From (6.7) it follows that

$$
\Omega_{t} \cap D\left(p_{0}, \varepsilon\right)=\Omega_{0} \cap D\left(p_{0}, \varepsilon\right) .
$$

The latter in its turn implies that $\left(C_{n}\right)$ is a null-chain for the the domain $\Omega_{t}$ as well and that for each $n \in \mathbb{N}$ the connected component of $\Omega_{t} \backslash C_{n}$ that contains $C_{n+1}$ coincides with $V_{n}$. It follows that the prime end of $\Omega_{t}$ defined by the null-chain $\left(C_{n}\right)$ is also degenerate and its impression is $\left\{p_{0}\right\}$. Hence there exists a point $\zeta_{t} \in \mathbb{T}$ such that the unrestricted limit $\lim _{z \rightarrow \zeta_{t}} F_{t}=p_{0}$ and the curve $F_{t}\left(\zeta_{t}[0,1)\right)$ tends to $P_{t}$. Again using rotation of $\mathbb{D}$ we can assume that $\zeta_{t}=1$.

Given a conformal mapping $F$ of the unit disk $\mathbb{D}$, the preimage of any null-chain for the domain $F(\mathbb{D})$ is a null-chain for $\mathbb{D}$. Therefore, for each $n \in \mathbb{N}$ the set $U_{0, n}:=F_{0}^{-1}\left(V_{n}\right)$ is a Jordan domain such that $\Gamma_{0, n}:=\mathbb{T} \cap \partial U_{0, n}$ is an arc and contains $\zeta=1$ as an internal point. By the same reason $U_{t, n}:=F_{t}^{-1}\left(V_{n}\right)$ is also a Jordan domain such that $\Gamma_{t, n}:=\mathbb{T} \cap \partial U_{t, n}$ is an arc, which contains $\zeta=1$ as an internal point. This means that the function $\Phi_{t}:=F_{t}^{-1} \circ F_{0}$ can be continuously extended to all internal points of the arc $\Gamma_{0,1}$ and that $\Phi_{t}\left(\Gamma_{0, n}\right)=\Gamma_{t, n}$ for all $n \in \mathbb{N}, n>1$. In particular, it follows that $\Phi_{t}$ extends analytically to the point $z=1$. Since all prime ends of $\mathbb{D}$ are degenerate,

$$
\bigcap_{n \in \mathbb{N}} \Gamma_{0, n}=\{1\}=\bigcap_{n \in \mathbb{N}} \Gamma_{t, n}
$$

Hence we have that $\Phi_{t}(1)=1$.

Summarizing the above facts one can easily conclude that $\Phi_{t} \in \tilde{\mathcal{C}}$. Therefore, assertion (ii) in Theorem 5.5 holds, which implies, according to this theorem, assertion (i). The chordal admissibility follows from assertion (ii) by Lemma 4.7. The proof is finished.

\section{An EXAMPLE}

Let us recall that a holomorphic function $\varphi: \mathbb{D} \rightarrow \mathbb{C}$ belongs to the disk algebra $\mathcal{A}$ if it has a continuous extension to the closed unit disk. In this section we construct an example 
of an evolution family $\left(\varphi_{s, t}\right)$ which is contained in the disk algebra, but has no associated Loewner chains $\left(f_{t}\right)$ with locally connected boundaries of the image domains $f_{t}(\mathbb{D})$. More precisely, $\partial f_{t}(\mathbb{D})$ is not locally connected for every $t \geq 0$ and each Loewner chain $\left(f_{t}\right)$ associated with $\left(\varphi_{s, t}\right)$. Another interesting property of the evolution family we construct below is as follows: $\left(\varphi_{s, t}\right)$ is a Goryainov-Ba (and in particular, chordal) evolution family and $\partial f_{t}(\mathbb{D})$ is not locally connected, because $f_{t}$ fails to have an angular limit at the point $z=1$.

Before we start constructing the announced example let us introduce some notations and results we are going to use.

As earlier we denote by $\mathrm{P}(D)$ the set of all prime ends of a simply connected domain $D$. Let $U$ and $W$ be simply connected domains and $F$ a conformal mapping of $U$ onto $W$. By $F^{\text {p.e. }}$ we will denote the bijective map between $\mathrm{P}(U)$ and $\mathrm{P}(W)$ induced by $F$. If $U$ is $\mathbb{H}$ or $\mathbb{D}$, we will identify $\mathrm{P}(U)$ with $\partial_{\infty} U$.

As in Section 5 we will denote by $\mathcal{H}$ the class of all univalent holomorphic selfmappings $G$ of $\mathbb{H}$ such that

$$
\lim _{\substack{z \rightarrow \infty \\ z \in \mathbb{H}}}(G(z)-z)=0
$$

Remark 7.1. The Riemann Mapping Theorem and the Prime End Theorem (see e.g. 40, p. 18]) imply:

(i) For any compact set $E \subset \mathbb{H} \cup \mathbb{R}$ there exists $G \in \mathcal{H}$ such that $G(\mathbb{H})=\mathbb{H} \backslash E$.

(ii) If $G, H \in \mathcal{H}$ and $G(\mathbb{H})=H(\mathbb{H})$, then $G=H$.

(iii) In particular, by assertion (ii), the function $G$ from assertion (i) is unique. We will denote it by $G[E]$. This function can be continued by symmetry to the lower half-plane and consequently can be represented in a neighbourhood of $\infty$ by a Laurent series with real coefficients and principal part of the form $a z+b, a>0$.

Definition 7.2. A Jordan arc $\Gamma$ with two different end-points $a_{1}$ and $a_{2}$ is said to be a slit in a domain $D$ if $\Gamma \subset \bar{D}:=D \cup \partial D$ and $\Gamma \cap \partial \mathbb{D}=\{a\}, a=a_{j}$ for $j=1$ or $j=2$. The point $a$ is called the root of the slit $\Gamma$ or its landing point. By parametrization of the slit $\Gamma$ we will mean a homeomorphic mapping $\gamma$ of a segment $[\alpha, \beta]$ onto $\Gamma$ with $\gamma(\beta) \in \partial D$.

The following result was proved in [30]. Closely connected results can be found in [42] and [3, Chapter IV§7, Theorem 2].

Theorem F. For any slit $\Gamma$ in the upper half-plane $\mathbb{H}$ landing at a finite point, there exists a parametrization $\gamma:[0, T] \rightarrow \mathbb{C}$ of $\Gamma$ such that the functions $\Phi_{0, t}:=G_{t}^{-1} \circ G_{0}$, where $G_{s}:=G[\gamma([s, T])], s \in[0, T]$, have the following Laurent expansion in a neighbourhood of $z=\infty$,

$$
\Phi_{0, t}(z)=z-\frac{t}{z}+\ldots
$$


Moreover, there exists a continuous function $\lambda:[0, T] \rightarrow \mathbb{R}$ such that for each $z \in \mathbb{H}$ the function $w(t):=\Phi_{0, t}(z), t \in[0, T]$, is a solution to the chordal Loewner equation

$$
\frac{d w(t)}{d t}=\frac{1}{\lambda(t)-w(t)}, \quad t \geq 0
$$

Now let us consider the domain $\Delta_{0}$ obtained by removing from the unit disk $\mathbb{D}$ the spiral curve $C:[0,+\infty) \rightarrow \mathbb{D}$ given by $C(\tau)=e^{i \tau}(1-1 /(\tau+2))$, tending asymptotically to the unit circle. Denote $\Delta_{\tau}:=\mathbb{D} \backslash C([\tau,+\infty))$. For each $\tau \in[0,+\infty)$ all the prime ends of the domain $\Delta_{\tau}$ are trivial (i.e., have impressions consisting of a unique point) except for exactly one prime end, impression of which coincides with the unit circle $\partial \mathbb{D}$. Denote this prime end by $P_{\tau}$. Let us denote by $F_{0}$ the conformal mapping of $\mathbb{H}$ onto $\Delta_{0}$ normalized by the conditions $F_{0}(i)=0, F_{0}^{p . e .}(\infty)=P_{0}$.

We claim that for each $\tau \geq 0$ there exists a conformal mapping $F_{\tau}$ from $\mathbb{H}$ onto $\Delta_{\tau}$ such that $\tilde{\Phi}_{0, \tau}:=F_{\tau}^{-1} \circ F_{0}$ belongs to $\mathcal{H}$. To see this, let us first consider the conformal mapping $H_{\tau}$ of $\mathbb{H}$ onto $\Delta_{\tau}$ normalized by the conditions $H_{\tau}(i)=0, H_{\tau}^{p . e .}(\infty)=P_{\tau}$. Further, the inverse mapping $H_{\tau}^{-1}$ extends continuously to the point $C(\tau)$. Hence the function $\Psi_{\tau}:=H_{\tau}^{-1} \circ F_{0}$ maps $\mathbb{H}$ onto $\mathbb{H}$ with slit along the Jordan curve $H_{\tau}^{-1}(C([0, \tau]))$ landing at the point on $H_{\tau}^{-1}(C(\tau)) \in \mathbb{R}$. Note also that $\Psi_{\tau}(\infty)=\infty$. By the Continuity theorem (see, e.g., [40, p. 18]), the map $\Psi_{\tau}$ is continuous up to the boundary. The argument of Remark 7.1 shows that in a neighbourhood of $\infty$ the function $\Psi_{\tau}$ has a Laurent expansion of the form

$$
\Psi_{\tau}(z)=a z+b+\frac{c_{1}}{z}+\ldots
$$

where $a>0$ and $b \in \mathbb{R}$. It follows that $F_{\tau}(z):=H_{\tau}(a z+b)$ is the desired conformal mapping.

Functions $\tilde{\Phi}_{0, \tau}$ have a Laurent expansion of the form

$$
\tilde{\Phi}_{0, \tau}=z-\frac{t(\tau)}{z}+\ldots
$$

where $t(\tau)>0$.

Now let us fix some $\sigma>0$. Using the connection between $F_{\sigma}$ and $H_{\sigma}$ we conclude that the function $F_{\sigma}^{-1} \circ F_{0}$ maps $\mathbb{H}$ onto $\mathbb{H} \backslash \Gamma$, where $\Gamma:=F_{\sigma}^{-1}(C([0, \sigma]))$ is a slit in $\mathbb{H}$ landing at $F_{\sigma}^{-1}(C(\sigma)) \in \mathbb{R}$. According to Theorem $\mathrm{F}$ there exists a parametrization $t \mapsto \gamma(t)$ of the slit $\Gamma$ such that functions $\Phi_{0, t}$ defined in the statement of this theorem satisfy (7.1). In particular, it means that for any $\tau \in[0, \sigma]$ there exists a unique $t_{\tau} \in[0, T]$ such that

$$
F_{\sigma}^{-1}(C(\tau))=\gamma\left(t_{\tau}\right), \quad \tau \in[0, \sigma]
$$

and the mapping $[0, \sigma] \ni \tau \mapsto t_{\tau} \in[0, T]$ is an increasing homeomorphism. Equality (7.4) implies that $\tilde{\Phi}_{0, \tau}=\Phi_{0, t_{\tau}}$. Consequently, by (7.1) and (7.3) $), t(\tau)=t_{\tau}$ for any $\tau \in[0, \sigma]$. Since $\sigma>0$ can be chosen arbitrarily, this means that $t \mapsto t(\tau)$ is a continuous strictly increasing mapping of $[0,+\infty)$ onto an interval of the form $\left[0, T_{0}\right), T_{0} \in(0,+\infty]$. 
Let us prove that $T_{0}=+\infty$. Suppose on the contrary that $T_{0}<+\infty$. Then it follows that $F_{\tau}$ converges, as $\tau \rightarrow+\infty$, uniformly on each compact subsets of $\mathbb{H}$. Indeed, denote $\tilde{\Phi}_{\nu, \mu}:=F_{\mu}^{-1} \circ F_{\nu}, \mu \geq \nu \geq 0$. Since the functions $F_{\tau}, \tau>0$, form a normal family in $\mathbb{H}$ and $\tilde{\Phi}_{\nu, \mu}(z)=z-(t(\mu)-t(\nu)) / z+\ldots$ in a neighbourhood of $z=\infty$, for any compact set $K \subset \mathbb{H}$ we have

$$
\begin{aligned}
\left|F_{\nu}(z)-F_{\mu}(z)\right|=\left|F_{\mu}\left(\tilde{\Phi}_{\nu, \mu}(z)\right)-F_{\mu}(z)\right| & \leq \\
& C_{K}^{o}\left|\tilde{\Phi}_{\nu, \mu}(z)-z\right| \leq C_{K}(t(\mu)-t(\nu)), \quad z \in K,
\end{aligned}
$$

provided $\mu>\nu$ and $\nu$ is large enough, say $\nu>\nu_{K}$, where in the last inequality we have applied that $\tilde{\Phi}_{\nu, \mu}$ belongs to $\mathfrak{P}_{0}$. Here $C_{K}^{o}, C_{K}$, and $\nu_{K}$ are positive constants independent of $\mu$ and $\nu$. This proves convergence of $F_{\tau}$ as $\tau \rightarrow+\infty$.

Denote the limit of $F_{\tau}$ by $F_{\infty}$. For each fixed $\nu \geq 0$ there exists also a limit $\tilde{\Phi}_{\nu, \infty}$ of $\tilde{\Phi}_{\nu, \mu}$ as $\mu \rightarrow+\infty$. Indeed, let $\mu_{2} \geq \mu_{1} \geq \nu$ and $w:=\tilde{\Phi}_{\nu, \mu_{1}}(z)$. Then

$$
\left|\tilde{\Phi}_{\nu, \mu_{2}}(z)-\tilde{\Phi}_{\nu, \mu_{1}}(z)\right|=\left|\tilde{\Phi}_{\mu_{1}, \mu_{2}}(w)-w\right| \leq \frac{\left(t\left(\mu_{2}\right)-t\left(\mu_{1}\right)\right)}{\operatorname{Im} w} \leq \frac{\left(t\left(\mu_{2}\right)-t\left(\mu_{1}\right)\right)}{\operatorname{Im} z} .
$$

Moreover, by [8, Theorem 4.2], $\tilde{\Phi}_{\nu, \infty} \in \mathfrak{P}_{0}$. Now we will prove that $F_{\infty}$ maps $\mathbb{H}$ onto the unit disk $\mathbb{D}$ in one-to-one manner. First of all we recall that $\cup_{\tau \geq 0} \Delta_{\tau}=\mathbb{D}$. Hence, for any point $w_{0} \in \mathbb{D}$ there exists $\nu \geq 0$ such that $F_{\nu}\left(z_{\nu}\right)=w_{0}$ for some $z_{\nu} \in \mathbb{H}$. Using the equality $F_{\nu}(z)=F_{\mu}\left(\tilde{\Phi}_{\nu, \mu}(z)\right), z \in \mathbb{H}$, we conclude that $z_{\mu}:=\tilde{\Phi}_{\nu, \mu}\left(z_{\nu}\right)$ solves the equation $F_{\mu}(z)=w_{0}$. Since $\tilde{\Phi}_{\nu, \mu}$ tends as $\mu \rightarrow+\infty$ to a non-constant function, the point $z_{\mu}$ tends to an internal point $z_{0} \in \mathbb{H}$. Taking into account locally uniform convergence of $F_{\mu}$ to $F_{\infty}$, we conclude that $F_{\infty}\left(z_{0}\right)=w_{0}$. This applies to any $w_{0} \in \mathbb{D}$. Hence, $F_{\infty}(\mathbb{H}) \supset \mathbb{D}$. On the other hand, the mapping $F_{\infty}$ is univalent and maps $\mathbb{H}$ into $\mathbb{D}$, because it is a locally uniform limit of univalent functions mapping $\mathbb{H}$ into $\mathbb{D}$ and is not a constant function. This proves that $F_{\infty}$ is a univalent mapping of $\mathbb{H}$ onto $\mathbb{D}$. Now we can pass to limits in the equality $F_{0}=F_{\tau} \circ \tilde{\Phi}_{0, \tau}$ in order to see that since $\tilde{\Phi}_{0, \infty}(z)$ tends to $\infty$ as $z$ tends to $\infty$ along the positive direction of the imaginary axis, the prime end $F_{0}^{\text {p.e. }}(\infty)$ contains a reachable point. This conclusion contradicts the construction, and hence, proves that $T_{0}=+\infty$.

Now let us define a family $\left(f_{t}: \mathbb{D} \rightarrow \mathbb{C}\right)_{t \geq 0}$ by the following relation

$$
f_{t(\tau)}=F_{\tau} \circ H, \quad H(\zeta):=i \frac{1+\zeta}{1-\zeta}, \quad \tau \geq 0
$$

Let $\varphi_{s, t}:=f_{t}^{-1} \circ f_{s}$. Note that $\Phi_{0, t}=H \circ \varphi_{0, t} \circ H^{-1}$ for all $t \in[0,+\infty)$. It follows that $\ell\left(\varphi_{s, t}\right)=t-s$ for all $0 \leq s \leq t<+\infty$. The family $\left(\varphi_{s, t}\right)$ satisfies EF1 and EF2. Therefore, Proposition 5.4 implies that $\left(\varphi_{s, t}\right)$ is a Goryainov-Ba evolution family of order $d=\infty$. Then, according to [17, Lemma 3.2], $\left(f_{t}\right)$ is one of the Loewner chains associated with this evolution family. 
Let us take any $\nu>0$ and $\mu \geq \nu$. Denote $s:=t(\nu), t:=t(\mu)$. Then the function $\varphi_{s, t}$ maps $\mathbb{D}$ onto $\mathbb{D} \backslash H^{-1}\left(F_{\mu}^{-1}(C([\nu, \mu]))\right)$. Again by the Continuity theorem (see e. g. [40, p. $18]$ ), we obtain that $\varphi_{s, t}$ belongs to the disk algebra $\mathcal{A}$.

However, for any $t \geq 0$ and $\tau$ such that $t=t(\tau)$, the prime end $f_{t}^{p . e .}(1)=P_{\tau}$ contains no reachable points. It follows that the angular limit of $f_{t}$ at the Denjoy-Wolff point $z=1$ of $\left(\varphi_{s, t}\right)$ does not exist for any $t \geq 0$. In particular, $f_{t}(\mathbb{D})$ is not locally connected.

Actually, the above statements hold for any Loewner chain $\left(g_{t}\right)$ associated with the evolution family $\left(\varphi_{s, t}\right)$. Assume on the contrary, that there exists a Loewner chain $\left(g_{t}\right)$ associated with $\left(\varphi_{s, t}\right)$ and $t \geq 0$ such that $g_{t}^{p . e .}(1)$ contains a reachable point, which we denote by $A$. Since by [17, Theorem 1.7] $g_{t}=h \circ f_{t}$ for some univalent holomorphic function $h: \mathbb{D} \rightarrow \mathbb{C}$, it would imply that there is a slit $\Gamma$ in $g_{t}(\mathbb{D})$ landing at the point $A \in$ $\partial g_{t}(\mathbb{D})=\partial h\left(f_{t}(\mathbb{D})\right) \subset \overline{h(\mathbb{D})}$ such that the curve $\Gamma_{0}:=h^{-1}(\Gamma)$ tends to the prime end $f_{t}^{p . e .}(1)$. By Theorem E, the curve $\Gamma_{0}$ lands at some particular point on the boundary of $f_{t}(\mathbb{D})$. This contradicts the fact that $f_{t}^{p . e .}(1)$ contains no reachable points.

Let us now summarize facts proved above in the following

Proposition 7.3. There exists a Goryainov-Ba evolution family $\left(\varphi_{s, t}\right), s \in[0,+\infty)$, $t \in[s,+\infty)$, of order $d=\infty$ such that

(i) $\ell\left(\varphi_{s, t}\right)=t-s$ for all $s \geq 0$ and $t \geq s$;

(ii) $\varphi_{s, t} \in \mathcal{A}$ for all $s \geq 0$ and $t \geq s$;

(iii) for each Loewner chain $\left(f_{t}\right)$ associated to the evolution family $\left(\varphi_{s, t}\right)$ and for any $t \geq 0$ the prime end $f_{t}^{\text {p.e. }}(1)$ is non-trivial and does not contain reachable points; in particular $\partial_{\infty} f_{t}(\mathbb{D})$ fails to be locally connected.

Remark 7.4. Using Theorem $\mathrm{F}$ one can write down the Herglotz vector field $G(z, t)$ corresponding to the evolution family $\left(\varphi_{s, t}\right)$ constructed in the above example. Namely,

$$
G(z, t)=(1-z)^{2} p(z, t), \quad p(z, t):=\frac{(1-u(t))(1-z)}{4(z-u(t))}, u(t):=H(\lambda(t)) \neq 1 .
$$

\section{Evolution FAMiLies In THE DisK ALGEBRA}

Proposition 7.3 shows that the local connectivity of $\partial_{\infty} f_{t}(\mathbb{D})$ for functions from a Loewner chain $\left(f_{t}\right)$ is not implied by the local connectivity of $\partial \varphi_{s, t}(\mathbb{D})$ for functions from the evolution family $\left(\varphi_{s, t}\right)$ generated by $\left(f_{t}\right)$. The following proposition shows that the converse implication is true.

Theorem 8.1. Let $\left(f_{t}\right)$ be a Loewner chain with associated evolution family $\left(\varphi_{s, t}\right)$. Suppose that $\partial_{\infty} f_{t}(\mathbb{D})$ is locally connected for all $t \geq 0$. Then $\varphi_{s, t}$ belongs to the disk algebra $\mathcal{A}$ for all $s \geq 0$ and $t \geq s$.

Proof. The argument of this proof follows the same ideas that the first two authors developed in [14. 
Fix $s<t$ and suppose that there is a point $a \in \partial \mathbb{D}$ where the function $\varphi_{s, t}$ has no continuous extension. Write $U_{n}=\{z \in \mathbb{D}:|z-a|<1 / n\}, n \in \mathbb{N}$. Then there exists a positive number $\varepsilon$ such that $\operatorname{diam}\left(\varphi_{s, t}\left(U_{n}\right)\right)>\varepsilon$ for all $n \in \mathbb{N}$, where $\operatorname{diam}(\Omega)$ denotes the Euclidean diameter of a given set $\Omega$. Since $\varphi_{s, t}\left(U_{n}\right)$ is connected, we can find Jordan arcs $C_{n}$ in $U_{n}$ such that $\operatorname{diam}\left(\varphi_{s, t}\left(C_{n}\right)\right)>\varepsilon$ for all $n \in \mathbb{N}$.

Moreover, since $\partial_{\infty} f_{s}(\mathbb{D})$ is locally connected, we have that

$$
\lim _{n \rightarrow+\infty} \operatorname{diam}^{\sharp}\left(f_{s}\left(C_{n}\right)\right)=0,
$$

where $\operatorname{diam}^{\sharp}(\Omega)$ denotes the spherical diameter of a given set $\Omega$. Since $f_{s}\left(U_{n+1}\right) \subset f_{s}\left(U_{n}\right)$ for all $n \in \mathbb{N}$, there is a point $c \in f_{s}(\mathbb{D}) \cup \partial_{\infty} f_{s}(\mathbb{D})$ such that

$$
\sup _{w \in f_{t}\left(\varphi_{s, t}\left(C_{n}\right)\right)} \chi(w, c)=\sup _{w \in f_{s}\left(C_{n}\right)} \chi(w, c) \leq \sup _{w \in f_{s}\left(U_{n}\right)} \chi(w, c) \underset{n \rightarrow \infty}{\longrightarrow} 0
$$

where $\chi(\cdot, \cdot)$ stands for the spherical distance.

Therefore, the arcs $\varphi_{s, t}\left(C_{n}\right)$ have diameter bigger than $\varepsilon$ for all $n \in \mathbb{N}$ and

$$
\lim _{n} \sup _{w \in f_{t}\left(\varphi_{s, t}\left(C_{n}\right)\right)} \chi(w, c)=0 .
$$

That is, $\left(\varphi_{s, t}\left(C_{n}\right)\right)_{n}$ is a sequence of Koebe arcs for the function $f_{t}$. This is a contradiction because a univalent function is normal (see, e.g., [39, Lemma 9.3 on p.262]), but by a theorem of Bagemihl and Seidel (see, e.g., [39, Corollary 9.1 on p. 267]) a non-constant normal function has no sequences of Koebe arcs. This completes the proof.

\section{ACKNOWLEDGEMENT}

The initial draft of this paper was prepared during a visit of the third author to the University of Seville, which was financially supported by the project MTM2006-14449C02-01. The third author would also like to thank the National Center for Theoretical Sciences, Hsinchu, Taiwan, where a substantial part of the work under the paper was carried out.

\section{REFERENCES}

[1] M. Abate, Iteration theory of holomorphic maps on taut manifolds, Mediterranean, Rende, 1989.

[2] N.I. Akhiezer and I.M. Glazman, Theory of linear operators in Hilbert space, Translated from the Russian and with a preface by Merlynd Nestell, Reprint of the 1961 and 1963 translations, Dover, New York, 1993.

[3] I.A. Aleksandrov, Parametric continuations in the theory of univalent functions (Russian), Izdat. "Nauka", Moscow, 1976.

[4] I.A. Aleksandrov, S.T. Aleksandrov and V.V. Sobolev, Extremal properties of mappings of a half plane into itself, in Complex analysis (Warsaw, 1979), 7-32, PWN, Warsaw.

[5] I.A. Aleksandrov and V.V. Sobolev, Extremal problems for certain classes of functions that are univalent in the half-plane, Ukrain. Mat. Ž. 22 (1970), 291-307.

[6] S.T. Aleksandrov, Parametric representation of functions univalent in the half plane, in Extremal problems of the theory of functions, 3-10, Tomsk. Gos. Univ., Tomsk, 1979. 
[7] S.T. Aleksandrov and V.V. Sobolev, Extremal problems in some classes of functions, univalent in the half plane, having a finite angular residue at infinity. Siberian Math. J. 27 (1986), no. 2, 145-154. Translation from Sibirsk. Mat. Zh. 27 (1986), no. 2, 3-13.

[8] R.O. Bauer, Chordal Loewner families and univalent Cauchy transforms, J. Math. Anal. Appl. 302 (2005), 484-501.

[9] F. Bracci, M.D. Contreras, and S. Díaz-Madrigal, Evolution Families and the Loewner Equation I: the unit disk, Preprint 2008. Available on ArXiv 0807.1594

[10] F. Bracci, M.D. Contreras, and S. Díaz-Madrigal, Evolution Families and the Loewner Equation II: complex hyperbolic manifolds, Math. Ann. 344 (2009), 947-962.

[11] L. de Branges, A proof of the Bieberbach conjecture, Acta Math. 154 (1985), 137-152.

[12] D. M. Burns and S. G. Krantz, Rigidity of holomorphic mappings and a new Schwarz lemma at the boundary, J. Amer. Math. Soc. 7 (1994), 661-676.

[13] E. F. Collingwood and A. J. Lohwater, The theory of cluster sets, Cambridge Univ. Press, Cambridge, 1966.

[14] M.D. Contreras and S. Díaz-Madrigal, Fractional iteration in the disk algebra: prime ends and composition operators, Revista Matemática Iberoamericana 21 (2005), 911-928.

[15] M.D. Contreras and S. Díaz-Madrigal, Analytic flows in the unit disk: angular derivatives and boundary fixed points, Pacific J. Math. 222 (2005), 253-286.

[16] M. D. Contreras, S. Díaz-Madrigal, and Ch. Pommerenke, On boundary critical points for semigroups of analytic functions, Math. Scand. 98 (2006), 125-142.

[17] M. D. Contreras, S. Díaz-Madrigal, and P. Gumenyuk, Loewner chains in the unit disk. To appear in Revista Matemática Iberoamericana; preprint availiable at arXiv:0902.3116v1 [math.CV]

[18] J.B. Conway, Functions of one complex variable, II. Second edition, Graduate Texts in Mathematics, 159. Springer-Verlag, New York-Berlin, 1996.

[19] W.F. Jr. Donoghue, Monotone matrix functions and analytic continuation, Springer-Verlag, New York - Heidelberg, 1974.

[20] P.L. Duren, Univalent functions, Springer, New York, 1983.

[21] G.M. Goluzin, Geometric theory of functions of a complex variable, Amer. Math. Soc., Providence, R.I., 1969. (translated from G. M. Goluzin, Geometrical theory of functions of a complex variable (Russian), Second edition, Izdat. "Nauka", Moscow, 1966)

[22] V.V. Goryainov, Semigroups of conformal mappings, Math. USSR Sbornik 57 (1987), 463-483.

[23] V.V. Goryainov, The Königs function and fractional integration of probability-generating functions (in Russian), Mat. Sb. 184 (1993), 55-74; translation in Russian Acad. Sci. Sb. Math. 79 (1994), $47-61$.

[24] V.V. Goryainov, The embedding of iterations of probability-generating functions into continuous semigroups (in Russian), Dokl. Akad. Nauk 330 (1993), 539-541; translation in Russian Acad. Sci. Dokl. Math. 47 (1993), 554-557.

[25] V.V. Goryaynov, Evolutionary families of analytic functions and time-nonhomogeneous Markov branching processes. (English. Russian original) Dokl. Math. 53 (1996), 256-258; translation from Dokl. Akad. Nauk 347 (1996), 729-731.

[26] V.V. Goryainov and I. Ba, Semigroups of conformal mappings of the upper half-plane into itself with hydrodynamic normalization at infinity, Ukrainian Math. J. 44 (1992), 1209-1217.

[27] B. Gustafsson and A. Vasil'ev, Conformal and potential analysis in Hele-Shaw cells, Birkhäuser, Basel, 2006.

[28] P.P. Kufarev, On one-parameter families of analytic functions (in Russian. English summary), Rec. Math. [Mat. Sbornik] N.S. 13 (55) (1943), 87-118. 
[29] P.P. Kufarev, On integrals of simplest differential equation with moving pole singularity in the righthand side, Tomsk. Gos. Univ. Uchyon. Zapiski 1 (1946), 35-48.

[30] P.P. Kufarev, V.V. Sobolev, and L.V. Sporyševa, A certain method of investigation of extremal problems for functions that are univalent in the half-plane, Trudy Tomsk. Gos. Univ. Ser. Meh.-Mat. 200 (1968), 142-164.

[31] G.F. Lawler, An introduction to the stochastic Loewner evolution, in Random Walks and Geometry, 261-293, Walter de Gruyter GmbH \& Co. KG, Berlin, 2004.

[32] G.F. Lawler, O. Schramm, and W. Werner, Values of Brownian intersection exponents. I. Half-plane exponents, Acta Math. 187 (2001), 237-273.

[33] G.F. Lawler, O. Schramm, and W. Werner, Values of Brownian intersection exponents. II. Plane exponents, Acta Math. 187 (2001), 275-308.

[34] G.F. Lawler, O. Schramm, and W. Werner, Values of Brownian intersection exponents. III. Twosided exponents, Ann. Inst. H. Poincaré Probab. Statist. 38 (2002), 109-123.

[35] G.F. Lawler, O. Schramm, and W. Werner, Conformal invariance of planar loop-erased random walks and uniform spanning trees, Ann. Probab. 32 (2004), 939-995

[36] K. Löwner, Untersuchungen über schlichte konforme Abbildungen des Einheitskreises, Math. Ann. 89 (1923), 103-121.

[37] I. Markina, D. Prokhorov, and A. Vasil'ev, Sub-Riemannian geometry of the coefficients of univalent functions, J. Funct. Anal. 245 (2007), 475-492.

[38] Ch. Pommerenke, Über dis subordination analytischer funktionen, J. Reine Angew Math. 218 (1965), $159-173$.

[39] Ch. Pommerenke, Univalent functions. With a chapter on quadratic differentials by Gerd Jensen, Vandenhoeck \& Ruprecht, Göttingen, 1975.

[40] Ch. Pommerenke, Boundary behaviour of conformal Maps, Springer-Verlag, Berlin, 1992.

[41] N.V. Popova, Investigation of some integrals of the equation $\frac{d w}{d t}=\frac{A}{w-\lambda(t)}$, Novosibirsk. Gos. Ped. Inst. Uchyon. Zapiski 8 (1949), 13-26.

[42] N.V. Popova, Dependence between Löwner's equation and the equation $\frac{d w}{d t}=\frac{1}{w-\lambda(t)}$, Izv. Akad. Nauk BSSR, Ser. Fiz.-Mat. Nauk 6 (1954), 97-98.

[43] D. Prokhorov and A. Vasil'ev, Univalent functions and integrable systems, Comm. Math. Phys. 262 (2006), 393-410.

[44] O. Schramm, Scaling limits of loop-erased random walks and uniform spanning trees, Israel J. Math. 118 (2000), 221-288.

[45] J.H. Shapiro, Composition operators and classical function theory, Springer-Verlag, New York, 1993.

[46] D. Shoikhet, Semigroups in geometrical function theory, Kluwer Academic Publishers, Dordrecht, 2001.

[47] A.G. Siskakis, Semigroups of composition operators on spaces of analytic functions, a review, in Studies on composition operators (Laramie, WY, 1996), 229-252, Contemp. Math., 213, Amer. Math. Soc., Providence, RI.

[48] V.V. Sobolev, Parametric representations for some classes of functions univalent in half-plane, Kemerov. Ped. Inst. Uchyon. Zapiski 23 (1970), 30-41.

[49] G. Valiron, Fonctions analytiques, Presses Univ. France, Paris, 1954.

[50] Yu.P. Vinogradov and P.P. Kufarev, On a problem of filtration, Akad. Nauk SSSR. Prikl. Mat. Meh. 12 (1948), 181-198. 
Camino de los Descubrimientos, s/n, Departamento de Matemática Aplicada II, Escuela Técnica Superior de Ingenieros, Universidad de Sevilla, Sevilla, 41092, Spain.

E-mail address: contreras@us.es

E-mail address: madrigal@us.es

Department of Mathematics, University of Bergen, Johannes Brunsgate 12, Bergen 5008, NORWAY.

E-mail address: Pavel.Gumenyuk@math.uib.no 